\title{
2 Pore pressure generation in a poro-elastic soil
}

\section{3 under moving train loads}

4 Xuecheng Bian ${ }^{\mathrm{a}}$, Jing $\mathrm{Hu}^{\mathrm{a}}$, David Thompson ${ }^{\mathrm{b}}$, William Powrie

5

6 Corresponding Author: Prof. David Thompson

7 bInstitute of Sound and Vibration Research, University of Southampton, University Road,

8 Highfield, Southampton SO17 1BJ, United Kingdom. Email: djt@isvr.soton.ac.uk

10 Co-author: Prof. Xuecheng Bian,

11 a Department of Civil Engineering, Key Laboratory of Soft Soils and Geoenvironmental

12 Engineering, MOE, Zhejiang University, Hangzhou, 310058, China.

13 Email: bianxc@zju.edu.cn

14

15 Co-author: Ms. Jing $\mathrm{Hu}$

16 aDepartment of Civil Engineering, Key Laboratory of Soft Soils and Geoenvironmental

17 Engineering, MOE, Zhejiang University, Hangzhou, 310058, China.

18 Email: jingh@zju.edu.cn

19

20 Co-author: Prof. William Powrie

$21{ }^{c}$ Engineering and Physical Sciences, University of Southampton, Highfield, Southampton.

22 SO17 1BJ United Kingdom. Email: W.Powrie@ soton.ac.uk 


\section{ABSTRACT:}

4 During the passage of a train along a railway track, the underlying soil experiences repeated

5 loading. If the soil is saturated, pore pressures will increase as the load passes, and these may

6 or may not start to dissipate before each load is removed. To investigate the dynamic

7 response and excess pore pressures generated in a saturated ground below the track, this

8 study uses a 2.5D finite element model (FEM) of a coupled track-embankment-ground

9 system. The saturated soil is modelled using Biot's theory of elastic wave propagation. The

10 implementation of the method is verified by comparison with semi-analytical solutions for

11 both single-phase elastic and poro-elastic media. It is then used to investigate the influence of

12 load speed $c$, soil Darcy permeability $k_{D}$ and stiffness on the excess pore water pressures

13 generated. It is found that the ratio $c / k_{D}$ determines the extent to which excess pore pressures

14 build up during passage of the load, at any given depth. For a saturated soil of a particular

15 stiffness, if $c / k_{D}$ is less than $10^{4}$, the soil can be viewed as highly permeable in relation to the

16 load speed and almost no excess pore pressure is developed. For a single moving load, there

17 is a critical value of $c / k_{D}$, above which the maximum pore pressure reaches a constant value;

18 this critical value depends on the depth. Below the critical value, the pore pressure

19 accumulated during the passage of a train depends on $c / k_{D}$ but is otherwise independent of the

20 load speed. The pore pressure accumulated during the passage of a bogie pair is greatest for

21 intermediate values of $c / k_{D}$. For small values of $c / k_{D}$ (high permeability), the pore pressure

22 build-up is small, whereas for large values of $c / k_{D}$ (low permeability) the pore pressure does

23 not dissipate during the loading cycle. The variation in the maximum stress ratio, $\left(\tau / \sigma^{\prime}\right)_{\max }$,

24 with permeability depends on the depth under consideration. The depth to which pore

25 pressures are generated and the effects of soil stiffness are also discussed in this paper. 
1 Keywords: Saturated porous media; Excess pore pressure; Soil permeability; High-speed

2 train; 2.5D FEM

\section{Introduction}

On a railway, the loads exerted by a train on the track and the underlying soil (the subgrade) are of short duration but cyclic and repeated. The dominant loading frequencies are that of a passing vehicle and its harmonics [1]. If the underlying soil is saturated and of lowto-moderate permeability, train passage will result in the generation of excess pore water pressures, which may or may not start to dissipate before each load is removed. Train loading will induce shear stresses in the soil, while excess pore pressures will tend to reduce the normal effective stress. The combined effect is to increase the ratio of shear to normal effective stress, and hence the effective (frictional) strength that the soil must mobilise to resist the applied loads. The mobilisation of additional strength will generate strains and displacements in the soil.

In classical soil mechanics, the problem would be investigated in the time domain using Terzaghi's theory of consolidation. However, the theory of elastic wave propagation in porous media, developed and originally applied to the analysis of ultrasonic geophysical test data by Biot [2] offers an alternative approach in which analysis is in the frequency domain. Biot's theory of elastic wave propagation considers the coupled motions of the solid elastic soil skeleton and the interstitial fluid.

Biot's original generalized formulation for low-frequency vibrations was recast in an alternative form that is more convenient for numerical work by Zienkiewicz and Shiomi [3].

23 Based on [3], Siddharthan et al. [4] developed two-dimensional (2D) semi-analytical

24 solutions by neglecting the relative motion between the solid and the fluid. Lu and Hanyga

25 [5], and Lu and Jeng [6] developed fundamental three-dimensional (3D) solutions for a 
1 layered porous half-space subjected to a moving vertical point load, which have become used

2 as benchmarks for the verification of various numerical solutions for moving load problems

3 on porous media. Xu et al. [7, 8] extended these solutions to an infinite beam on a layered

4 poro-elastic half-space subjected to moving loads. To improve the calculation efficiency of a

5 full 3D analysis, a 2.5D approach has been developed and used [9-12] to determine the

6 response of poro-elastic media.

7

Using these $2 \mathrm{D} / 2.5 \mathrm{D} / 3 \mathrm{D}$ solutions, several researchers have brought insights into the

8 problem of the dynamic response of poro-elastic media. Lefeuve-Mesgouez and Mesgouez [13]

9 studied the response due to a moving rectangular load on a poroelastic halfspace. The results

10 showed that, as the load speeds approach or exceed a critical value, significant dynamic

11 amplification or resonance effects occur. There are differences in the vibration amplitude between a poro-elastic medium and its single-phase equivalent, as shown in [9, 14]; the vertical displacement of the poroelastic soil medium is smaller than that of an elastic soil at low speed loads, but larger than that of an elastic soil when the load speed approaches the critical value. Jin et al. [15] used a 2D model to study the stresses and excess pore pressures in a poroelastic half-space due to a moving line load. They showed that at high speeds, stresses in the poroelastic medium are smaller than in an elastic solid. investigated rail vibration and soil stresses. In [16] the pore water pressure variation with

20 depth below the track was presented for different non-dimensional parameter combinations. It was shown that the load speed can magnify the pore pressure, but has limited influence on the affected depth. Theodorakopoulos [18] and Theodorakopoulos et al. [19] carried out a parametric study of a moving load on a $2 \mathrm{D}$ poroelastic medium, and found that the effect of porosity and permeability on the pore pressure response is more pronounced in soft materials, especially at high load speeds. 
The Biot theory has recently been applied in the study of the dynamic response of

2 saturated soil around tunnels. Yuan et al. [20] investigated the dynamic response due to a

3 load moving in a tunnel modelled in $2 \mathrm{D}$. This also showed critical velocity effects. An

4 analytical model of a tunnel in a full space [21] showed that the pore pressure increases as the

5 soil permeability decreases and as the load speed increases. Di et al. [22, 23] investigated the

6 stress and pore water pressure distribution under a tunnel, including its variation with depth.

7 In reality, the behaviour of the soil may not be purely elastic or the peak pore pressure

8 may start to dissipate; hence it is possible that some residual excess pore pressure (or suction)

9 may be present after a train has passed. There is therefore a potential concern about the

10 timescale of excess pore pressure dissipation relative to the speed or frequency of application

11 of individual loads within a train. In previous studies, the timescales of pore pressure

12 generation and dissipation and their relationship with the soil permeability are rarely

13 discussed.

14 In this research, a 2.5D FEM model based on Biot's theory of elastic wave propagation

15 in a poro-elastic medium is used to investigate the dynamic response and excess pore

16 pressures generated in a saturated ground below one or more moving loads. The aim of this

17 paper is to investigate (i) the influence of the soil permeability and stiffness, in relation to the

18 train speed, on the excess pore water pressures generated; and (ii) the loaded depth (i.e., the

19 depth to which significant increases in stress and pore pressure may be generated) during a

20 train passage. In Section 2 the modelling method is briefly introduced and in Section 3 its

21 implementation is validated by comparison with analytical solutions. Section 4 discusses

22 results for a single moving load and Section 5 for a train of moving loads. 


\section{2. Modelling method}

\section{$2 \quad 2.12 .5 D$ finite element modelling of saturated embankment-ground system}

3 The constitutive equations for a homogeneous porous medium (Biot [24]) take the form

$$
\begin{gathered}
\sigma_{i j}=2 \mu \varepsilon_{i j}+\lambda \delta_{i j} e-\alpha \delta_{i j} p \\
p=-\alpha M-M w_{i, i} \\
e=u_{i, i}
\end{gathered}
$$

4 where $u_{i}$ denotes the average displacement of the solid and $w_{i}$ the displacement of the pore

5 fluid relative to the solid skeleton; $\varepsilon_{i j}$ and $e$ are the strain tensor and the dilation of the solid

6 skeleton; $\sigma_{i j}$ is the total stress; $p$ is the excess pore pressure (over and above the equilibrium,

7 usually hydrostatic, value); $\delta_{i j}$ is the Kronecker delta; $\lambda$ and $\mu$ are the Lamé constants of the

8 equivalent bulk solid ( $\mu$ is the shear modulus, also denoted $G_{s}, \lambda=\frac{E_{s} \cdot v}{(1+v) \cdot(1-2 v)}$, where $E_{s}$ is

9 the Young's modulus, and $v$ is the Poisson's ratio); $\alpha$ and $M$ are the Biot parameters $\left(\alpha=K / K_{s}\right.$

10 and $\frac{1}{M}=\frac{n}{K_{f}}+\frac{\alpha-n}{K_{S}}$, in which $K$ is the bulk modulus of the assembly, $K_{s}$ is the bulk modulus of

11 the solid grains, $K_{f}$ is the bulk modulus of the pore fluid, and $n$ is the porosity). In Eq. (1) the

12 summation convention is used and , $i$ indicates differentiation by the spatial coordinate $x_{i}$.

13 The rate of relative fluid discharge per unit total area in the $i(i=x, y, z)$ direction, $q_{i}$, is

14 given by

$$
q_{i}=\frac{\partial w_{i}}{\partial t}
$$

15 where $t$ is time.

16 The equations of motion for bulk porous medium and a unit volume of pore fluid under

17 low frequency load can be expressed in $u-w$ form

$$
\begin{gathered}
\mu u_{i, j j}+\left(\lambda+\alpha^{2} M+\mu\right) u_{j, j i}+\alpha M w_{j, j i}=\rho_{b} \ddot{u}_{i}+\rho_{f} \ddot{w}_{i} \\
\alpha M u_{j, j i}+M w_{j, j i}=\rho_{f} \ddot{u}_{i}+m \ddot{w}_{i}+\frac{\rho_{f} g}{k_{D}} \dot{w}_{i}
\end{gathered}
$$


where $\rho_{b}$ is the bulk density of the porous medium and $\rho_{f}$ is the density of the pore fluid. $\rho_{b}$ and $\rho_{f}$ are related by the expression $\rho_{b}=(1-n) \rho_{s}+n \rho_{f}$, where $\rho_{s}$ is the density of the soil grains (grain density). $g$ is gravitational acceleration and $k_{D}$ is the Darcy permeability of the porous medium; $m$ represents the effective density of the fluid when moving relative to the solid skeleton and is defined as $m=\frac{a_{\infty} \rho_{f}}{n}$, where $a_{\infty}$ is the tortuosity. A superimposed dot above a variable denotes a time derivative.

Biot's governing equations - Eqs (3) and (4) - are solved using the Fourier transform of $u(x, y, z, t)$ with respect to the $x$-coordinate and time $t$, which is defined by:

$$
\tilde{\bar{u}}\left(\xi_{x}, y, z, \omega\right)=\int_{-\infty}^{+\infty} \int_{-\infty}^{+\infty} u(x, y, z, t) e^{i \xi_{x} x} e^{-i \omega t} d x d t
$$

9 The corresponding inverse transform with respect to $\xi_{x}$ and $\omega$ is

$$
u(x, y, z, t)=\frac{1}{4 \pi^{2}} \int_{-\infty}^{+\infty} \int_{-\infty}^{+\infty} \tilde{u}\left(\xi_{x}, y, z, \omega\right) e^{-i \xi_{x} x} e^{i \omega t} d \xi_{x} d \omega
$$

where $(y, z)$ denote the horizontal (transverse) and vertical coordinates normal to the $x$ -

11 direction, $\omega$ is the circular frequency and $\xi_{x}$ the horizontal wavenumber ( $2 \pi$ divided by

12 wavelength) relevant to the $x$-direction. ' - ' and ' $\sim$ ' above a variable indicate components in

13 the wavenumber and frequency domains, respectively.

Yuan et al. [12] give the specific steps of the 2.5D solution process. The cross-section of the domain is discretized into finite elements and solutions in the wavenumber domain are obtained by the conventional finite element method, which takes the form:

$$
\begin{gathered}
\left(\mathbf{K}_{1}+\mathbf{K}_{2}-\mathbf{M}_{1}\right) \widetilde{\widetilde{\mathbf{U}}}+\left(\mathbf{L}_{1}-\mathbf{M}_{2}\right) \tilde{\overline{\mathbf{W}}}=\tilde{\overline{\mathbf{F}}} s \\
\left(\mathbf{K}_{3}-\mathbf{M}_{3}\right) \widetilde{\mathbf{U}}+\left(\mathbf{L}_{2}-\mathbf{M}_{4}\right) \widetilde{\mathbf{W}}=\tilde{\overline{\mathbf{F}}} f
\end{gathered}
$$

17 in which $\mathbf{M}$ are mass matrices, $\mathbf{K}$ and $\mathbf{L}$ are stiffness matrices, and $\mathbf{U}, \mathbf{W}$ are vectors of the

18 solid displacement and the displacement of the pore fluid, respectively at nodal positions. $\mathbf{F}^{s}$ 
1 and $\mathbf{F}^{f}$ are equivalent nodal load vectors. The detailed expressions for $\mathbf{M}, \mathbf{K}, \mathbf{L}$ are given in

2 Appendix A.

3 To prevent wave reflection back into the model from the boundaries of the finite element

4 domain, the multilayer damped boundary approach described by Liu and Jerry [25] is

5 adopted. The elements near the boundary are divided into $j$ element sets as shown in Fig. 1. In

6 the model, the damped boundary consists of 4-noded, 2.5D finite elements of the same type

7 as other elements. In the multilayer damped boundary approach, damping is added to the

8 elements increasingly towards the boundary.

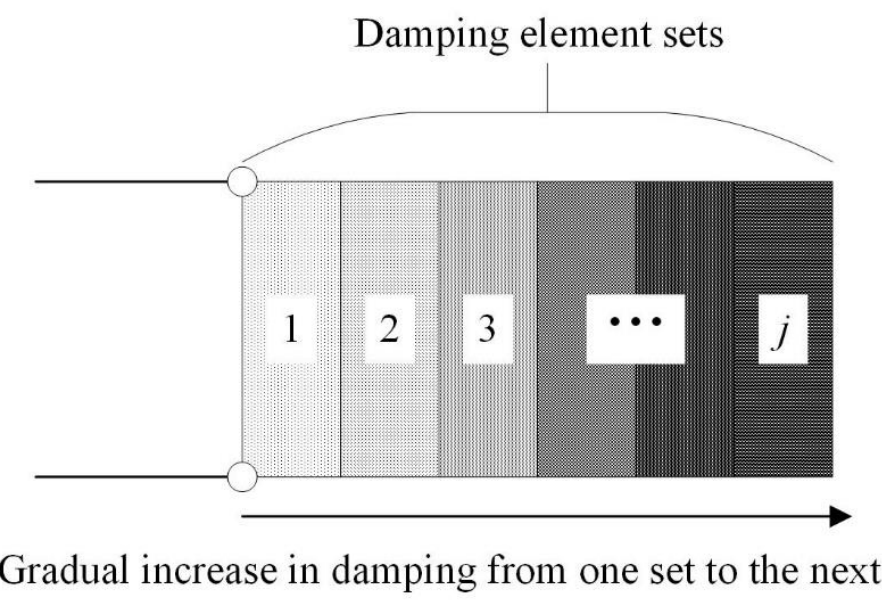

Gradual increase in damping from one set to the next

Figure 1. Artificial damping boundary section

To ensure that the level of damping increases gradually towards the boundary, the

12 Young's modulus for the $k$ th element set is expressed as:

$$
E_{k}=E+\mathrm{i} D_{0} \zeta^{k} E, \quad k=0,1,2, \ldots, j
$$

13 where $E$ is the real part of the Young's modulus, $D_{0}$ is the initial material loss factor

14 and $\zeta(\zeta>1)$ is a constant. To determine an appropriate value of $\zeta$ requires an iterative

15 procedure, in which $\zeta$ is increased until the responses obtained for two cases show no significant differences. This approach prevents a sudden increase in the damping, which

17 would itself cause reflection of the propagating wave. It simulates the wave-absorbing effect

18 of the soil beyond the finite element domain, so that the ground in the model will behave as a 
1 semi-infinite medium (half space). Although other methods are available, e.g. [9, 10, 12], this method has the advantage that it does not need any new formulation and is easy to program. fluid movement in or out of the model. A 'drainage boundary' is realized by setting the permeability of elements at the boundary to be very high to ensure that the dynamic pore

6 pressure tends to zero at the boundary.

\subsection{Modelling the track}

A schematic diagram of the track-embankment-ground model used in this study is shown in Fig. 2. The railway structure and the underlying embankment are aligned in the $x$ direction. Along with the ground, they are assumed to be infinite and their properties invariant in this direction. The track has a contact width $B$ with the embankment. The two rails are modelled together as a single Euler beam with combined mass per unit length $m_{r}$ and bending stiffness $E I_{r}\left(\mathrm{~N} \cdot \mathrm{m}^{2}\right)$. The sleepers are modelled as a distributed mass $m_{s}$ per unit length of track. The rails and sleepers are connected by rail pads, which are modelled as a distributed vertical spring of stiffness $k_{p}(\mathrm{~N} / \mathrm{m} / \mathrm{m})$ and damping $c_{p}(\mathrm{Ns} / \mathrm{m} / \mathrm{m})$ per unit length of track.

The governing equation for the vertical motion of the rail beam due to a single moving harmonic load in the wavenumber and frequency domain is:

$$
E I_{r} \xi_{x}^{4} \tilde{\bar{u}}_{r}-m_{r} \omega^{2} \tilde{\bar{u}}_{r}+\left(k_{p}+i \omega c_{p}\right)\left(\tilde{\bar{u}}_{r}-\tilde{\bar{u}}_{s}\right)=\tilde{\bar{F}}_{r}\left(\xi_{x}, \omega\right)
$$

19 where $u_{r}$ is the rail displacement, $u_{s}$ is the displacement of the sleepers, $F_{r}$ is the interaction

20 force (excited by the train) at the rail surface moving with a speed $c$, and $\delta$ is the Dirac delta 21 function.

22 For the sleepers,

$$
-m_{s} \omega^{2} \tilde{\bar{u}}_{s}+\left(k_{p}+i \omega c_{p}\right)\left(\tilde{\bar{u}}_{s}-\tilde{\bar{u}}_{r}\right)=-F_{s}=F_{e}
$$


1 where $F_{s}$ is the interaction force per unit length between the embankment and sleepers, $F_{e}$ is

2 the support force at the embankment surface.

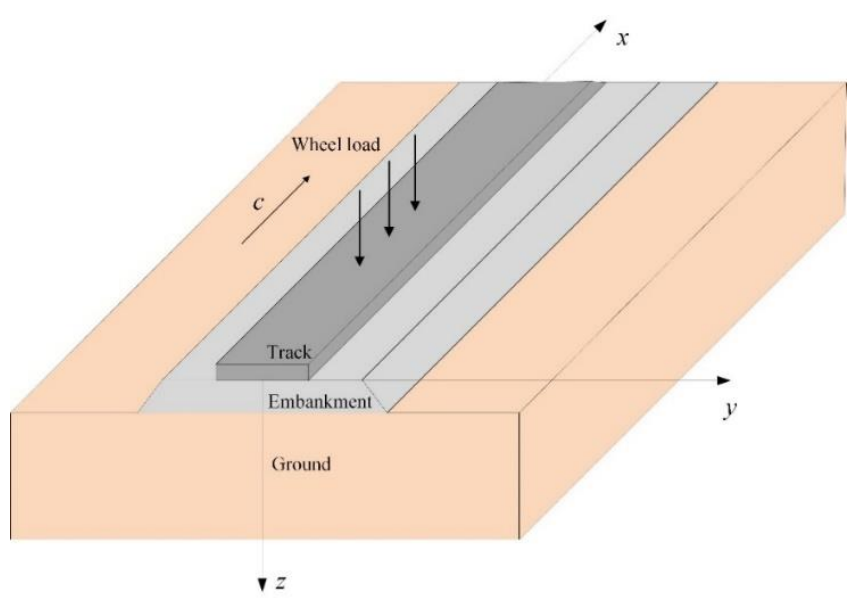

5 (a)

6

7

11

13

14

15

16

17

18

19

\section{(a)}

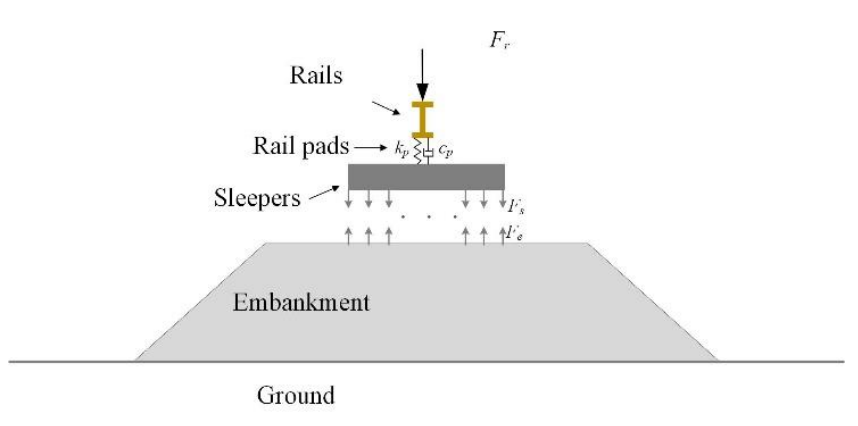

(b)

Figure 2. Diagram of track-embankment-foundation model: (a) track-embankment-ground system; (b) track details

\subsection{Mathematical description of the forces on the rails}

The passage of a train is represented by a series of moving axle loads corresponding to the train geometry in Fig. 3.

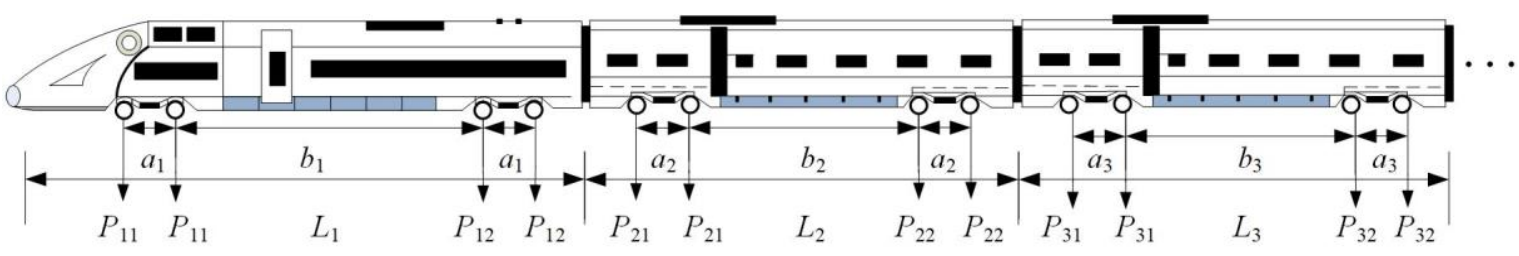

Figure 3. Geometric profile of train wheel loads 
1 For a train comprising $R$ vehicles, successive axle loads moving with a constant velocity $c$

2 can be expressed by the model of Takemiya and Bian [26]:

$$
\tilde{\bar{F}}_{r}\left(\xi_{x}, \omega\right)=\sum_{n=1}^{R} \tilde{\bar{P}}_{n}\left(\xi_{x}, \omega\right)
$$

3 The detailed expression for $P_{n}$, representing the wheel loads of the $n$th vehicle, is

$$
\tilde{\bar{P}}_{n}\left(\xi_{x}, \omega\right)=\sum_{i=1}^{4}\left(e^{-i \xi_{x} x_{n i}}\right) \tilde{\bar{P}}_{n i}\left(\xi_{x}, \omega\right)
$$

4 in which $x_{n 1}=\sum_{n=1}^{R} L_{n}+x_{0} ; x_{n 2}=a_{n}+\sum_{n=1}^{R} L_{n}+x_{0} ; x_{n 3}=a_{n}+b_{n}+\sum_{n=1}^{R} L_{n}+x_{0}$;

$5 \quad x_{n 4}=2 a_{n}+b_{n}+\sum_{n=1}^{R} L_{n}+x_{0}$ where $P_{n 1}, P_{n 2}, P_{n 3}$ and $P_{n 4}$ define the axle loads for the

6 four wheelsets of the $n$th vehicle, respectively, $L_{n}$ is the length of the $n$th vehicle, $x_{0}$ is the

7 distance from a reference position to the first axle load position, $a_{n}$ is the bogie wheelbase

8 and $b_{n}$ is the distance between the second and third axles of the vehicle of the $n$th vehicle, as

9 indicated in Fig. 3.

\section{$10 \quad 2.4$ Coupling of track structure and ground motions}

11 From Eq. (9) and (10), by eliminating $\tilde{\bar{u}}_{r}$, the track motion under axle loading can be

12 written as:

$$
K_{T} U_{s}=F_{e}+F_{P}
$$

13 where $F_{e}$ is the support force from the embankment-surface, $F_{P}$ contains the applied force at

14 the rail surface, and $K_{T}$ denotes the track dynamic stiffness. $U_{s}$ is a scalar containing only the sleeper displacement $\tilde{\bar{u}}_{s}$, which is equal to the displacement $\tilde{\bar{u}}$ of the points at the embankment surface. The expressions for $K_{T}$ and $F_{P}$ are:

$$
K_{T}=\frac{\left(E I_{r} \xi_{x}{ }^{4}-m_{r} \omega^{2}+k_{p}+i \omega c_{p}\right)\left(-m_{s} \omega^{2}+k_{p}+i \omega c_{p}\right)-\left(k_{p}+i \omega c_{p}\right)^{2}}{E I_{r} \xi_{x}^{4}-m_{r} \omega^{2}+k_{p}+i \omega c_{p}}
$$




$$
F_{P}=\frac{\left(k_{p}+i \omega c_{p}\right)}{E I_{r} \xi_{x}^{4}-m_{r} \omega^{2}+k_{p}+i \omega c_{p}} \tilde{\bar{F}}_{r}\left(\xi_{x}, \omega\right)
$$

2 The track is coupled to the ground model by adding the track stiffness $K_{T}$ to the stiffness

3 matrix in Eq.(7) in the vertical direction, eliminating $F_{e}$.

4

3. Validation of the 2.5D FEM model

In this section, the dynamic response of the ground subjected to a moving load is investigated using 2.5D FE numerical modelling and compared with reference solutions, first for an elastic half space and then for a saturated medium. A single-layer foundation model (width $150 \mathrm{~m} \times$ depth $80 \mathrm{~m}$; Fig.4), with a constant point load of $1 \mathrm{~N}$ moving at a speed $c$ applied at the surface was used to verify the 2.5D FEM. The numerical results for an observation point at $(x, 1,1)$ have been used for model verification.

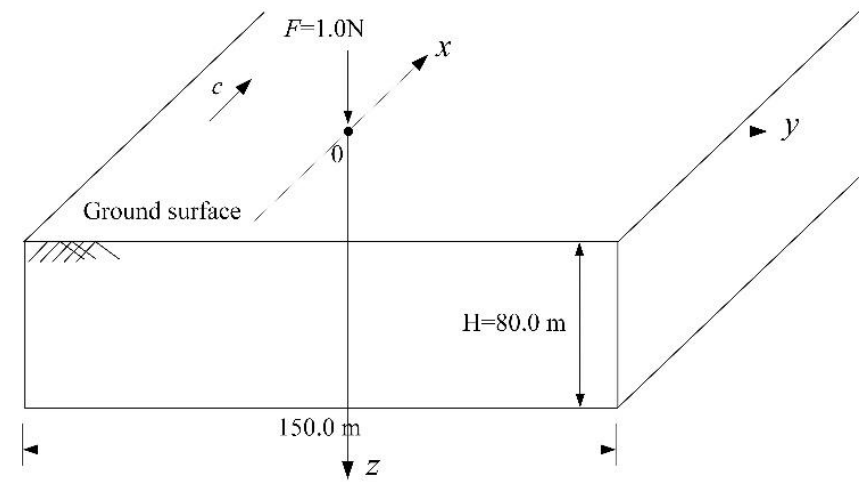

13 Figure 4. Verification model

\subsection{Comparison with semi-analytical solution for a single-phase elastic medium}

The parameters used to characterise the elastic medium were taken from [6], to facilitate comparison with their results for verification purposes (Table 1). A saturated, two-phase medium may be reduced to a single-phase elastic material by setting $\alpha, M, \rho_{f}, n$ and $m$ to approximately zero, as indicated. 
1 Table 1. Parameters used to model the elastic medium in validation analyses (after Lu and

2 Jeng [6])

\begin{tabular}{ll}
\hline Biot parameter $\alpha$ & 0.001 \\
Biot parameter $M$ & $0.001 \mathrm{MPa}$ \\
Young's modulus of solid grain $E_{s}$ & $56.25 \mathrm{MPa}$ \\
Shear modulus of solid grain $G_{s}$ & $25 \mathrm{MPa}$ \\
Poisson's ratio $v$ & 0.125 \\
Soil grain density $\rho_{s}$ & $2500 \mathrm{~kg} / \mathrm{m}^{3}$ \\
Fluid density $\rho_{f}$ & $0.001 \mathrm{~kg} / \mathrm{m}^{3}$ \\
Porosity $n$ & 0.001 \\
Darcy permeability $k_{D}$ & $1 \times 10^{-9} \mathrm{~m} / \mathrm{s}$ \\
Effective density m & $0.001 \mathrm{~kg} / \mathrm{m}^{3}$ \\
\hline
\end{tabular}

3

4

Fig. 5 shows that the calculated dynamic vertical displacements at $(x, 1,1)$ in response to

5 a unit point load moving at a speed of $75 \mathrm{~m} / \mathrm{s}$ are in close agreement with the semi-analytical

6 solutions for a homogeneous elastic half-space determined using the thin layer element

7 method [27].

8

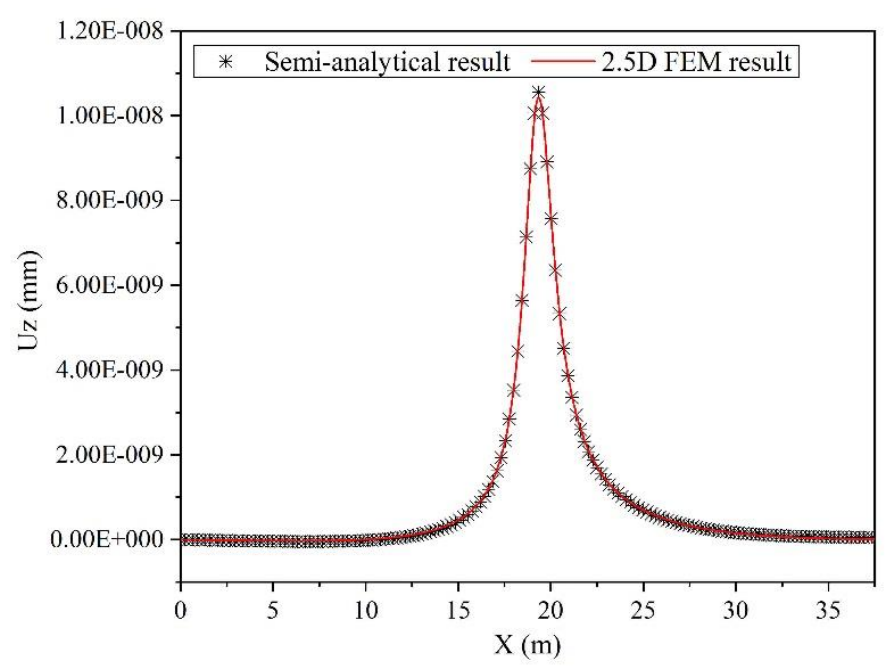

9 Figure 5. Dynamic vertical displacement at $(0,1,1)$ for an elastic medium. 


\section{$1 \quad 3.2$ Verification of 2.5D FEM for saturated medium}

2 The properties used to model a saturated soil are given in Table 2. These parameters were

3 again taken from [6], which is used as a basis for comparison.

4 Table 2. Parameters used to model a saturated medium in validation analyses (after Lu and 5 Jeng [6])

\begin{tabular}{ll}
\hline Biot parameter $\alpha$ & 0.95 \\
Biot parameter $M$ & $5000 \mathrm{MPa}$ \\
Young's modulus of bulk solid $E$ & $6744.6 \mathrm{MPa}$ \\
Shear modulus of bulk solid $G$ & $2997.6 \mathrm{MPa}$ \\
Poisson's ratio $v$ & 0.125 \\
Soil grain density $\rho_{s}$ & $2500 \mathrm{~kg} / \mathrm{m}^{3}$ \\
Fluid density $\rho_{f}$ & $1000 \mathrm{~kg} / \mathrm{m}^{3}$ \\
Porosity $n$ & 0.3 \\
Darcy permeability $k_{D}$ & $1 \times 10^{-6} \mathrm{~m} / \mathrm{s}$ \\
Effective density $m$ & $6670 \mathrm{~kg} / \mathrm{m}^{3}$ \\
\hline
\end{tabular}

6

$7 \quad$ The dynamic vertical displacements and pore pressures at the point $(x, 1,1)$ for a unit $(1$

$8 \mathrm{~N}$ ) single load $F$ moving at a speed $c=121 \mathrm{~m} / \mathrm{s}$ are shown in Fig. 6 . The displacements are

9 normalised by multiplying by $\frac{G a_{R}}{F}$, with $F=1 \mathrm{~N}, a_{R}=1.0 \mathrm{~m}$, while the pore pressures are

10 normalised by multiplying by $\frac{a_{R}^{2}}{F}$. Both are in close agreement with the semi-analytical

11 results given in [6]. 


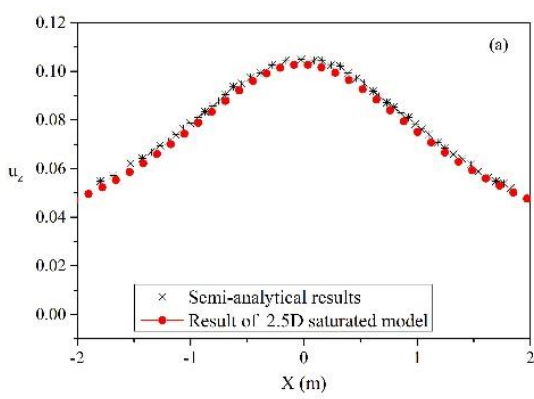

(a)

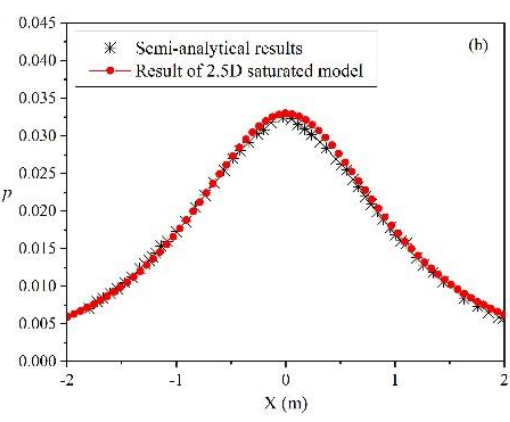

(b)

3 Figure 6. Dynamic response at $(0,1,1)$ in saturated medium for a unit load: (a) vertical

4 dynamic displacement, normalised by multiplying by $G a_{R} / F$; (b) excess pore pressure

$5 \quad$ normalised by multiplying by $a_{R}^{2} / F\left(a_{R}=1.0 \mathrm{~m}\right)$.

\section{4. Numerical results for a single moving load}

8 In this section, the response of a saturated ground to a single moving load on the track is

9 determined. The cross-section of the track-embankment-ground model is shown

schematically in Fig. 7. The embankment was $0.3 \mathrm{~m}$ high and treated as a single-phase elastic

11 solid, i.e. the generation of pore water pressures in this region was not considered. The

12 ground was modelled as a single layer of saturated soil of thickness $20 \mathrm{~m}$. The overall depth

13 of this model was $50 \mathrm{~m}$ from the ground surface and its width $100 \mathrm{~m}$. Layers of higher permeability, shown shaded in Fig. 7, were located both above and below the saturated stratum over the full width of the mesh. The thickness of the upper layer was $0.3 \mathrm{~m}$. The thickness of the bottom layer was $29.7 \mathrm{~m}$ in the initial model; this could be changed simply by setting the permeability of a different depth within the saturated stratum to be the same as that of the bottom layer.

19 According to the Biot theory, no water flows into or out of any element. The high permeability layers are used to simulate drainage boundaries by ensuring that the dynamic

21 pore pressure at the boundary is zero. All the finite elements, including the elastic 
1 embankment, saturated ground and drainage layer, were of the same type - poro-elastic with

26 degrees of freedom per node. By setting $\alpha, M, \rho_{f}, n$ and $m$ approximately equal to zero, a

3 saturated, two-phase medium may be reduced to a single-phase elastic material, as in the

4 verification in Fig. 5; this was used to model the embankment.

5

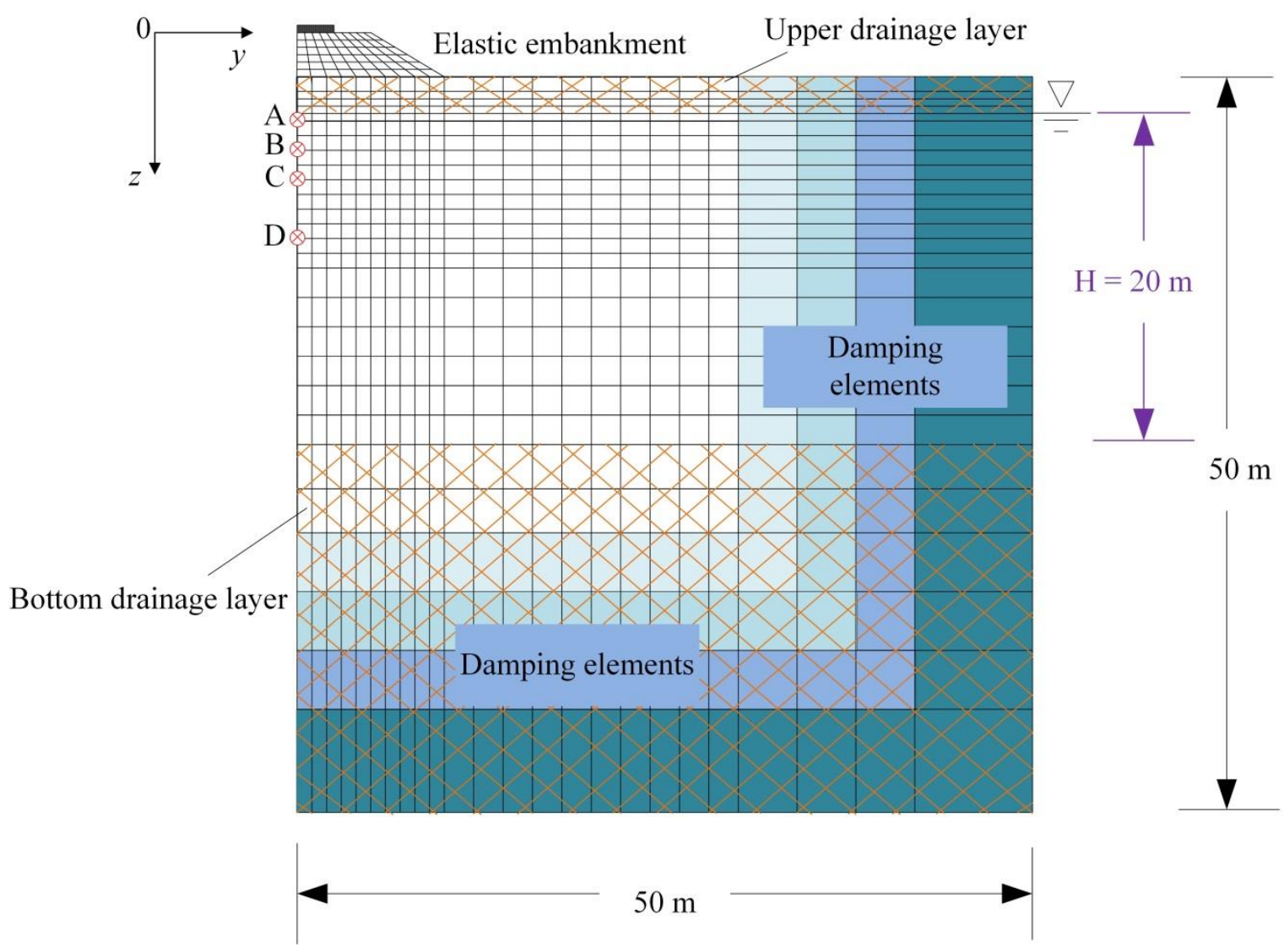

7 Figure 7. Schematic view of half of the finite element model of the track and saturated

8 ground (not to scale)

9 The maximum element size in the central area (40 m wide $\times 30 \mathrm{~m}$ deep) of the mesh in

10 Fig. 7 is $0.5 \mathrm{~m} \times 0.5 \mathrm{~m}$; at the right and left hand boundaries the maximum element size

11 increases to $2 \mathrm{~m} \times 2 \mathrm{~m}$. With depth, the element size increases from $0.05 \mathrm{~m}$ to $0.1,0.2,0.5$,

$12 \quad 1.0$ and then $2.0 \mathrm{~m}$. The total thickness of the damped layers at the edges and bottom of the

13 model is $20 \mathrm{~m}$. 
1 The parameters used to represent the embankment, the saturated ground and the drainage

2 layers are summarised in Table 3; those for the track are listed in Table 4. The shear wave

3 velocity of the saturated soil was $121.7 \mathrm{~m} / \mathrm{s}$, which is within the range of possible values for

4 soils such as clays. For the value of Poisson's ratio adopted (0.35), the Rayleigh wave

5 velocity of the saturated soil is $92 \%$ of the shear wave velocity, i.e. $112 \mathrm{~m} / \mathrm{s}$. The ground was

6 assigned a nominal value of damping loss factor of 0.05 to avoid numerical issues.

7 Calculations were carried out for a range of values of Darcy permeability $k_{D}$.

9 moving at $100 \mathrm{~m} / \mathrm{s}$ was passed along the surface of the above, full model and also of a

10 reduced model of half width $30 \mathrm{~m}$ rather than $50 \mathrm{~m}$. The displacement results were found to

11 be identical.

13 Table 3. Parameters used for the embankment, saturated ground and drainage layers

\begin{tabular}{llll}
\hline Soil layer & Embankment & Saturated ground & Drainage layer \\
Biot parameter $\alpha$ & 0.001 & 1.0 & 1.0 \\
Biot parameter $M$ & 0.001 & $1930 \mathrm{MPa}$ & $1930 \mathrm{MPa}$ \\
Young's modulus of bulk solid $E$ & $160 \mathrm{MPa}$ & $108 \mathrm{MPa}$ & $453 \mathrm{MPa}$ \\
Shear modulus of bulk solid $G$ & $64 \mathrm{MPa}$ & $40 \mathrm{MPa}$ & $168 \mathrm{MPa}$ \\
Poisson's ratio $v$ & 0.25 & 0.35 & 0.35 \\
Soil grain density $\rho_{s}$ & $2500 \mathrm{~kg} / \mathrm{m}^{3}$ & $2700 \mathrm{~kg} / \mathrm{m}^{3}$ & $2700 \mathrm{~kg} / \mathrm{m}^{3}$ \\
Fluid density $\rho_{f}$ & 0.001 & $1000 \mathrm{~kg} / \mathrm{m}^{3}$ & $1000 \mathrm{~kg} / \mathrm{m}^{3}$ \\
Soil damping loss factor $D_{0}$ & 0.05 & 0.05 & 0.05 \\
Porosity $n$ & 0.001 & 0.4 & 0.4 \\
Darcy permeability $k_{D}$ & $1 \times 10^{-9} \mathrm{~m} / \mathrm{s}$ & $1 \times 10^{-3} \sim 1 \times 10^{-9} \mathrm{~m} / \mathrm{s}$ & $1 \mathrm{~m} / \mathrm{s}$ \\
Effective density $m$ & 0.001 & $2500 \mathrm{~kg} / \mathrm{m}^{3}$ & $2500 \mathrm{~kg} / \mathrm{m}^{3}$ \\
\hline
\end{tabular}

14

15 Table 4. Parameters used to model the track (for two rails)

\begin{tabular}{ll}
\hline Mass of rail beam per unit length of track $m_{r}$ & $120 \mathrm{~kg} / \mathrm{m}$ \\
Bending stiffness of rail beam $E I_{r}$ & $13.24 \mathrm{MNm}{ }^{2}$ \\
Mass of sleeper per unit length of track $m_{s}$ & $490 \mathrm{~kg} / \mathrm{m}$ \\
Rail pad stiffness per unit length of track $k_{p}$ & $270 \mathrm{MN} / \mathrm{m} / \mathrm{m}$ \\
\hline
\end{tabular}




\begin{tabular}{ll}
\hline Rail pad damping per unit length of track $c_{p}$ & $83.5 \mathrm{kNs} / \mathrm{m} / \mathrm{m}$ \\
Contact width of railway and foundation $B$ & $2.4 \mathrm{~m}$
\end{tabular}

Throughout this section, the calculated response is for a single moving load $(72.25 \mathrm{kN})$;

3 this corresponds to the weight of one quarter of a vehicle. Five different train speeds

$4(c=1 \mathrm{~m} / \mathrm{s}, 3 \mathrm{~m} / \mathrm{s}, 10 \mathrm{~m} / \mathrm{s}, 30 \mathrm{~m} / \mathrm{s}$ and $100 \mathrm{~m} / \mathrm{s})$ and seven different values of soil permeability

5 (Darcy permeability $k_{D}=10^{-3} \mathrm{~m} / \mathrm{s}, 10^{-4} \mathrm{~m} / \mathrm{s}, 10^{-5} \mathrm{~m} / \mathrm{s}, 10^{-6} \mathrm{~m} / \mathrm{s}, 10^{-7} \mathrm{~m} / \mathrm{s}, 10^{-8} \mathrm{~m} / \mathrm{s}, 10^{-9} \mathrm{~m} / \mathrm{s}$ )

6 were considered. These speeds were not intended to be realistic, but follow a logarithmic

7 progression for comparison over the range of permeabilities. Four observation points, marked

8 A, B, C and D in Fig. 7, were chosen, at depths below the top of the embankment of $0.7 \mathrm{~m}$,

$9 \quad 1.2 \mathrm{~m}, 1.6 \mathrm{~m}$ and $2.6 \mathrm{~m}$, respectively.

\subsection{Time history of pore water pressures}

Fig. 8 shows time history curves for the excess pore water pressure at point A, which is located just $0.1 \mathrm{~m}$ below the upper drainage layer. Results are shown for all values of soil permeability and two values of train speed $(c=3 \mathrm{~m} / \mathrm{s}$ and $c=30 \mathrm{~m} / \mathrm{s})$. When the soil permeability is high $\left(k_{D}=10^{-3} \mathrm{~m} / \mathrm{s}\right.$ or $\left.10^{-4} \mathrm{~m} / \mathrm{s}\right)$, the pore pressure changes very little at the lower speed ( $c=3 \mathrm{~m} / \mathrm{s}$, Fig. $8(\mathrm{a})$ ). This is consistent with the soil being free-draining, so that any excess pore water pressure is able to dissipate almost as soon as it develops. When the soil permeability is very low $\left(k_{D}=10^{-8} \mathrm{~m} / \mathrm{s}\right.$ or $\left.10^{-9} \mathrm{~m} / \mathrm{s}\right)$, the dynamic pore pressure is much greater and is essentially symmetrical about the time of maximum load application. This is consistent with the permeability of the soil being so low that there is no opportunity for excess pore pressure dissipation during the time the load being is applied. As the permeability

21 is increased above $k_{D}=10^{-8} \mathrm{~m} / \mathrm{s}$, the maximum induced pore water pressure reduces but the response curve becomes asymmetrical, with a negative pore pressure being developed ahead 
1 of the moving load. This is also seen in other solutions to the moving load problem, obtained

2 using different methods $[16,17,22]$.

3 Increasing the train speed (Fig. 8(b)) makes little difference to the magnitude or pattern of

4 pore pressure generation when the soil permeability is low $\left(k_{D}=10^{-8} \mathrm{~m} / \mathrm{s}\right.$ or $\left.10^{-9} \mathrm{~m} / \mathrm{s}\right)$.

5 However, increasing the train speed increases the numerical value of permeability needed for

6 a soil to be considered highly permeable, in the sense that no transient pore water pressure is

7 generated. This is consistent with the reduction in the time available for excess pore pressure

8 dissipation during the period of application of the load. In Fig. 8(b), the results for a Darcy

9 permeability of $10^{-3} \mathrm{~m} / \mathrm{s}$ correspond to those at the lower speed for $k_{D}=10^{-4} \mathrm{~m} / \mathrm{s}$; similarly,

10 for other values of permeability, these results suggest that changes in speed and permeability

11 are interchangeable.

12 The time histories plotted in this paper only show part of the calculated response. The

13 2.5D method uses a certain wavenumber resolution $\mathrm{d} \xi_{x}$, here between approximately $10^{-3}$ and

$1410^{-2} \mathrm{rad} / \mathrm{m}$, which defines the effective model length as $2 \pi / \mathrm{d} \xi_{x}$. Thus in the present analyses the model length is at least $600 \mathrm{~m}$. Differences in the pore pressure seen before and after the passage of the load dissipate over the remaining time of the calculation.

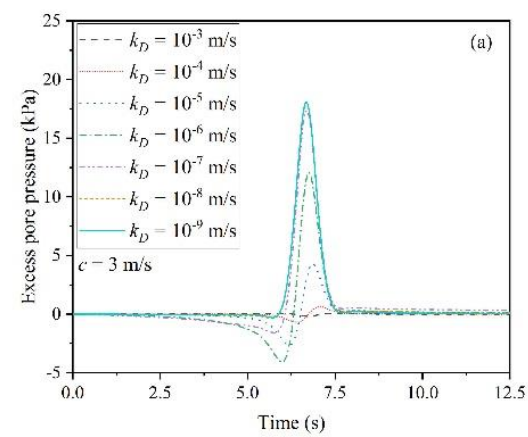

(a) $c=3 \mathrm{~m} / \mathrm{s}$

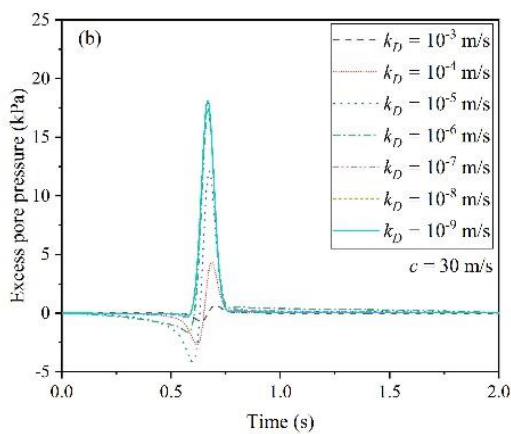

(b) $c=30 \mathrm{~m} / \mathrm{s}$

Figure 8 . Time history curve of excess pore pressure at point $\mathrm{A}(z=0.7 \mathrm{~m})$ for train speeds of

(a) $3 \mathrm{~m} / \mathrm{s}$ and (b) $30 \mathrm{~m} / \mathrm{s}$ at permeabilities ranging from $10^{-3} \mathrm{~m} / \mathrm{s}$ to $10^{-9} \mathrm{~m} / \mathrm{s}$. 
Fig. 9 shows the distribution of maximum excess pore pressure with depth when the load

2 is directly above the observation point. At a given load speed, the maximum excess pore

3 pressure generated reduces as the soil permeability increases, as already explained. The depth

4 at which the maximum excess pore pressure occurs increases slightly with increasing soil

5 permeability. This is consistent with the excess pore pressures that would have been

6 generated in the soil nearer the upper drainage layer having been able to dissipate during

7 application of the load. For the same reasons, an increase in the load speed causes an increase

8 in the maximum pore pressure and raises the level at which it occurs. In these analyses the

9 lower drainage layer is at a depth of $z=20.6 \mathrm{~m}$, which is sufficient not to have an influence:

10 the excess pore pressure never rises above $0.1 \mathrm{kPa}$ at $10 \mathrm{~m}$ depth, owing to the spread of the

11 applied load laterally to the side of and along the track.

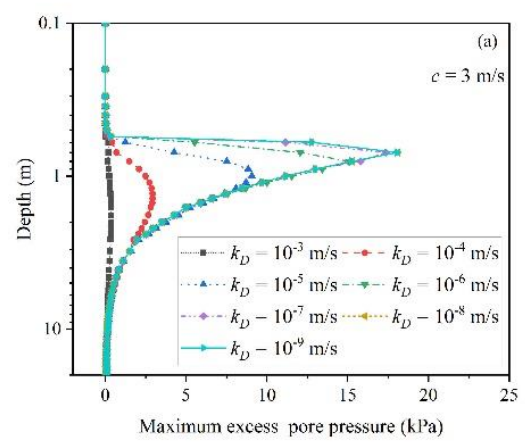

$13 \quad$ (a) $c=3 \mathrm{~m} / \mathrm{s}$

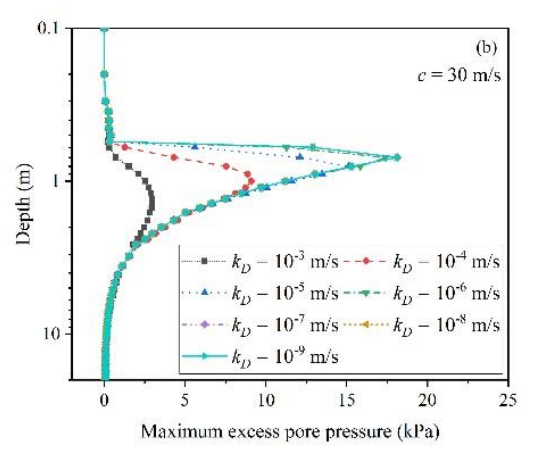

(b) $c=30 \mathrm{~m} / \mathrm{s}$

14 Figure 9. Excess pore pressure versus depth for train speeds of (a) $3 \mathrm{~m} / \mathrm{s}$ and (b) $30 \mathrm{~m} / \mathrm{s}$ at 15 permeabilities ranging from $10^{-3} \mathrm{~m} / \mathrm{s}$ to $10^{-9} \mathrm{~m} / \mathrm{s}$.

\subsection{Effect of velocity and permeability}

The investigations described in the previous section focused mainly on the effects of load speed for single values of soil permeability. The combined effects of train speed and soil permeability may be illustrated by considering the dimensionless ratio $c / k_{D}$. It has already been seen that variations in $c$ and $k_{D}$ appear interchangeable. Fig. 10 shows the dependence of 
1 maximum excess pore pressure on $c / k_{D}$ at different depths: results of analyses at different

2 train speeds are denoted by different symbols. The effective stress is also shown; this is the

3 total stress minus the pore pressure, Eq. (1).

4 Fig. 10 shows that the dependence of the maximum excess pore pressure and effective 5 stress on $c / k_{D}$ does not vary with the load speed, apart from a small increase at $c=100 \mathrm{~m} / \mathrm{s}$;

6 this is caused by the proximity to the Rayleigh wave speed of the saturated ground (which is

7 about $111 \mathrm{~m} / \mathrm{s}$ ). Fig. 10 also shows that there is a critical value of $c / k_{D}$ for the development of

8 excess pore pressure and effective stress, such that when $c / k_{D}$ is smaller than the critical

9 value, the maximum excess pore pressure increases, while the maximum effective stress

10 decreases, with increasing $c / k_{D}$. Above the critical value, the maximum excess pore pressure

11 and effective stress remain independent of $c / k_{D}$. To identify the critical value of $c / k_{D}$ the

12 changes in pore pressure are investigated: if the change in pore pressure between two

13 adjacent values of $c / k_{D}$ was less than $5 \%$, then the smaller of these values of $c / k_{D}$ was taken to

14 be the critical value. The same critical value of $c / k_{D}$ applies for the results at $100 \mathrm{~m} / \mathrm{s}$, i.e.

15 close to the Rayleigh wave speed, even though the magnitude of the pore pressure is greater.

16 In Fig.10, the total stress, determined as the sum of the effective stress and the pore

17 pressure in accordance with normal soil mechanics principles, is constant at any depth for

18 load speeds less than $100 \mathrm{~m} / \mathrm{s}$. The total stress reduces with depth owing to load dissipation

19 effects. At load speeds in excess of $100 \mathrm{~m} / \mathrm{s}$, the total stress increases as the critical velocity is

20 approached. 
3 Figure 10. Dependence of maximum excess pore pressure and effective stress on $c / k_{D}$ at (a)

(a) 5 from $10^{-3} \mathrm{~m} / \mathrm{s}$ to $10^{-9} \mathrm{~m} / \mathrm{s}$.

6

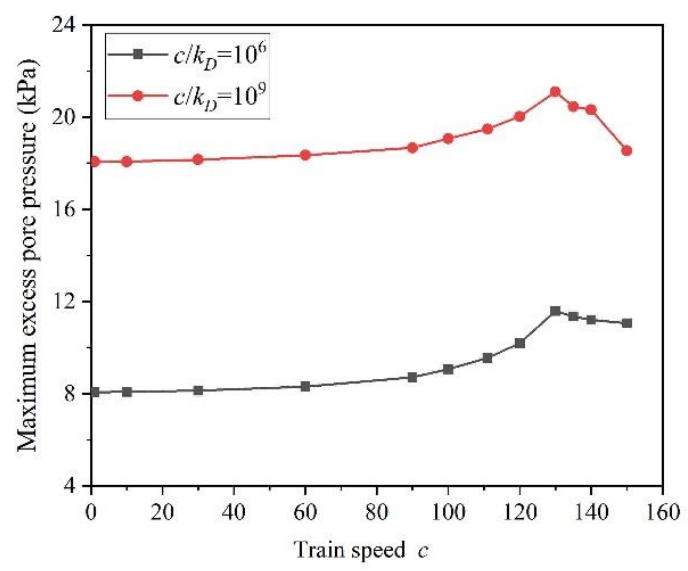

(1)

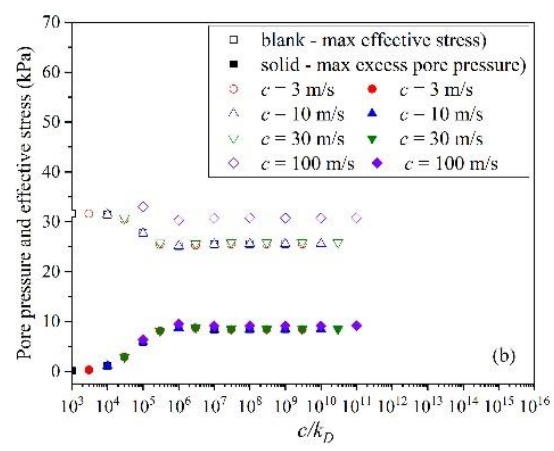

(b)

point $\mathrm{A}, z=0.7 \mathrm{~m}$ and $(\mathrm{b})$ point $\mathrm{B}, z=1.2 \mathrm{~m}$ for various train speeds at permeabilities ranging

7 Figure 11. Maximum excess pore pressure plotted as a function of train speed for two

8 example values of the parameter $c / k_{D}$.

9 Fig. 11 shows the maximum excess pore pressure as a function of train speed for two

10 example values of the parameter $c / k_{D}$. Fig. 11 shows that the critical speed of this model (i.e.

11 the speed with maximum response) is about $130 \mathrm{~m} / \mathrm{s}$, which is higher than the Rayleigh wave

12 speed of the saturated soil owing to the presence of the stiffer embankment and drainage

13 layer. The maximum displacement results (not shown) follow the same trend as the maximum

14 excess pore pressure. For a given value of $c / k_{D}$, the result only varies with the load speed 
1 when the critical speed is approached. This is caused by the increased soil deflections as the

2 load speed approaches the wave speed in the soil.

3 The critical value of $c / k_{D}$ observed in Fig. 10 changes substantially with depth. At $z=$

$40.7 \mathrm{~m}$, the critical value is $10^{8}$; for $z=1.2 \mathrm{~m}$, it is $10^{6}$. Fig. 12 shows the how the critical

5 value of $c / k_{D}$, derived from graphs like Fig. 10, decreases with depth; the rate of change at

6 shallow depths is particularly large. Thus, the excess pore pressure at shallow depths will be

7 potentially much more sensitive to changes in load speed or soil permeability: this is at least

8 in part a result of proximity to the drainage layer.

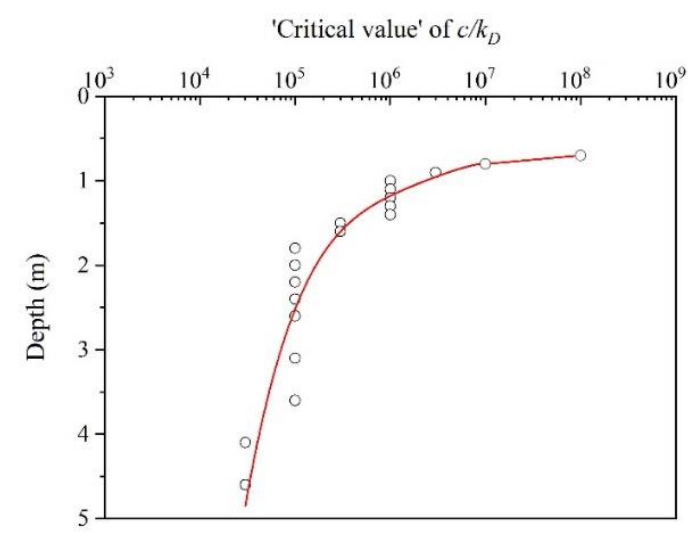

9

10 Figure 12. Variation in the critical value of $c / k_{D}$ with depth $z$

11 In Fig. 9, negative pore pressures were noted ahead of the load. Fig. 13 shows the

12 dependence of the maximum negative excess pore pressure (suction) on $c / k_{D}$ : the relationship

13 is the same for all load speeds investigated. The maximum negative excess pore pressure

14 tends to zero when $c / k_{D}$ reaches its critical value given in Figure 12. 


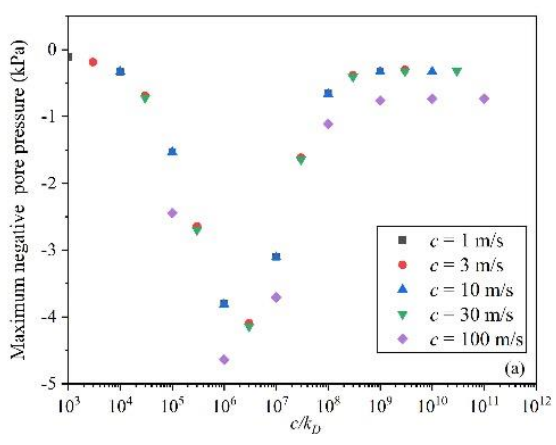

2

(a)

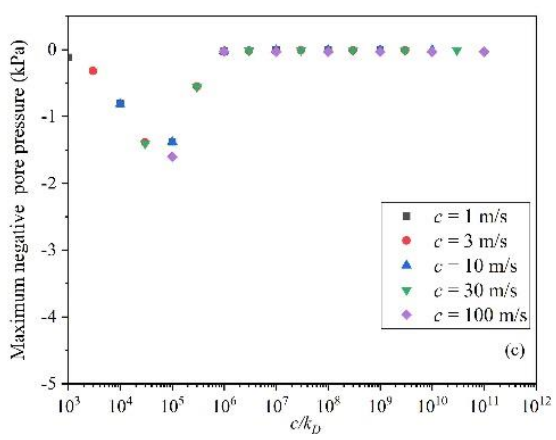

3

4

(c)

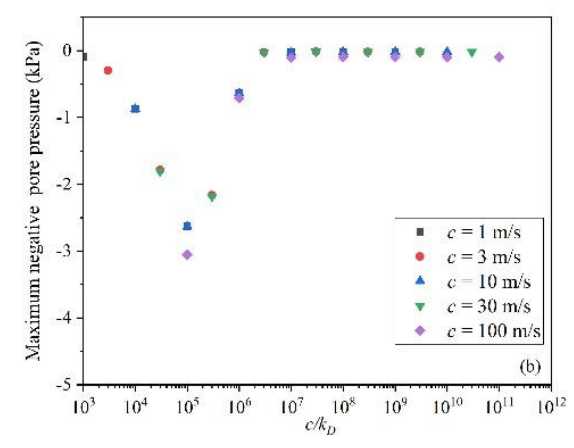

(b)

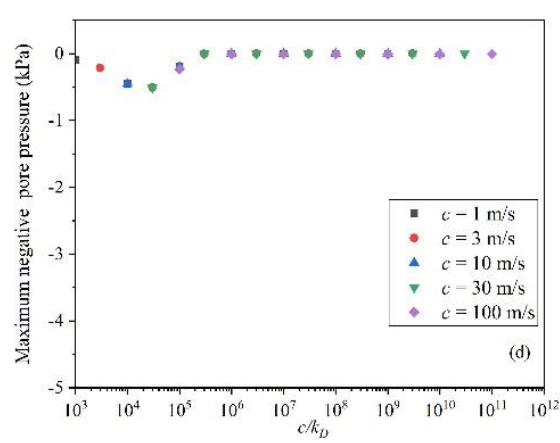

(d)

5 Figure 13. Dependence of maximum negative excess pore pressure (suction) on $c / k_{D}$ at

6 different depths: (a) point $\mathrm{A}, z=0.7 \mathrm{~m}$, (b) point $\mathrm{B}, z=1.2 \mathrm{~m}$, (c) point $\mathrm{C}, z=1.6 \mathrm{~m}$, (d) point $7 \quad \mathrm{D}, z=2.6 \mathrm{~m}$

\subsection{Stress ratio}

9 Excess pore pressures will tend to reduce the normal effective stress, see Eq. (1); hence the

10 generation of excess pore pressures will increase the ratio of shear to normal effective stress.

11 Fig. 14 shows time history curves for the normal effective stress, i.e. effective stress plus

12 body force, and the shear stress at point $\mathrm{A}$, depth $\mathrm{z}=0.7 \mathrm{~m}$, for soil permeabilities ranging

13 from $10^{-3}$ to $10^{-7} \mathrm{~m} / \mathrm{s}$ at a train speed of $10 \mathrm{~m} / \mathrm{s}$. 


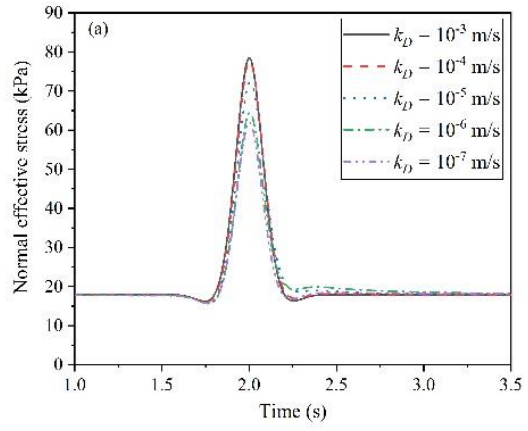

(a)

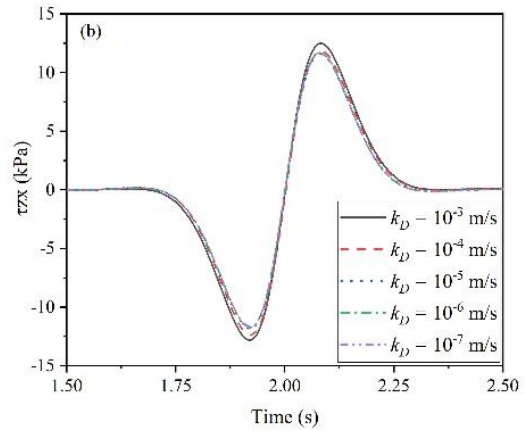

(b)

3 Figure 14. Time history curve of normal effective stress and shear stress at point $\mathrm{A}, z=0.7 \mathrm{~m}$

4 (a) normal effective stress and (b) shear stress for a train speed of $10 \mathrm{~m} / \mathrm{s}$ at permeabilities

5 ranging from $10^{-3} \mathrm{~m} / \mathrm{s}$ to $10^{-7} \mathrm{~m} / \mathrm{s}$.

6 When the soil permeability is high $\left(k_{D}=10^{-3} \mathrm{~m} / \mathrm{s}\right.$ or $\left.10^{-4} \mathrm{~m} / \mathrm{s}\right)$, the normal effective stress

7 change is greater (Fig. 14(a)). This is consistent with the fact that when the permeability of

8 the soil is high there is no opportunity for excess pore pressure build-up. In this case the loads

9 are carried by the soil skeleton during the time over which they are applied, rather than by the

10 fluid. The shear stress is zero when the normal effective stress is greatest (i.e., when the load

11 is directly above the observation point). The maximum shear stress also increases slightly

12 with increasing permeability (Fig. 14(b)), but not as significantly as the maximum normal

13 effective stress - and, of course, the two are out of phase.

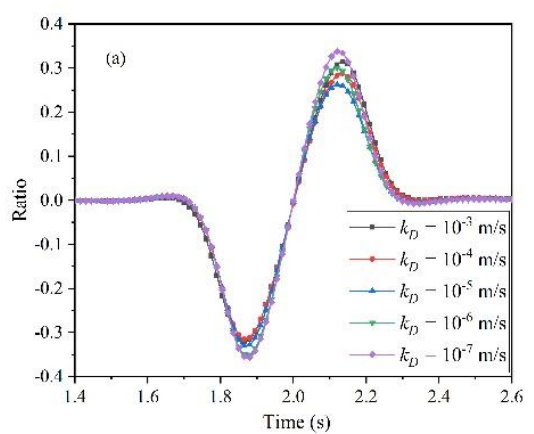

14

15

(a)

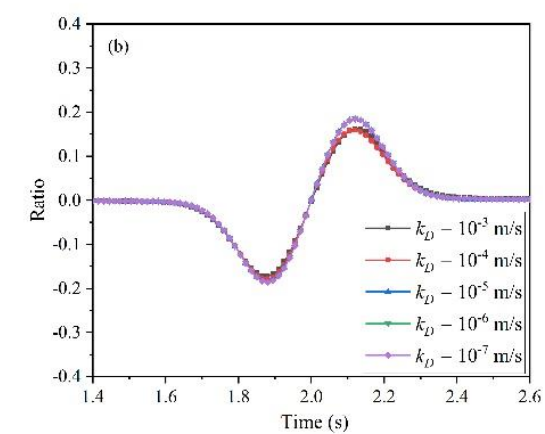

(b) 
1 Figure 15 . Time history curves of stress ratio $\left(\tau / \sigma^{\prime}\right)$ at (a) point $\mathrm{A}, z=0.7 \mathrm{~m}$ and (b) point $\mathrm{B}, z$

$2=1.2 \mathrm{~m}$ for a train speed of $10 \mathrm{~m} / \mathrm{s}$ and permeabilities ranging from $10^{-3} \mathrm{~m} / \mathrm{s}$ to $10^{-7} \mathrm{~m} / \mathrm{s}$.

3 As explained in the introduction, the generation of excess pore water pressures will

4 increase the ratio of shear to normal effective stress, and hence the strength that the soil must

5 mobilise to resist the applied loads. Fig. 15 shows time history curves of the stress ratio ( $\left.\tau / \sigma^{\prime}\right)$

6 (i.e., the ratio of shear stress to normal effective stress) at two depths, $z=0.7 \mathrm{~m}$ and $z=1.2$

$7 \mathrm{~m}$, for soil permeabilities ranging from $10^{-3}$ to $10^{-7} \mathrm{~m} / \mathrm{s}$ and a train speed of $10 \mathrm{~m} / \mathrm{s}$. The

8 maximum value of this stress ratio may be comparable with the yield value of clay (typically

9 at least $\tan 20^{\circ}$ or 0.36 [28-30]), and changes only slightly with the permeability. At a depth

10 of $0.7 \mathrm{~m}$, the maximum value falls as the soil permeability is reduced from $10^{-3}$ to $10^{-5} \mathrm{~m} / \mathrm{s}$,

11 but then increases again with a further reduction in soil permeability to $10^{-7} \mathrm{~m} / \mathrm{s}$. At $1.2 \mathrm{~m}$

12 depth, the maximum value is similar for all soil permeabilities shown except $10^{-7} \mathrm{~m} / \mathrm{s}$, for

13 which a higher maximum stress ratio is calculated.

14

15

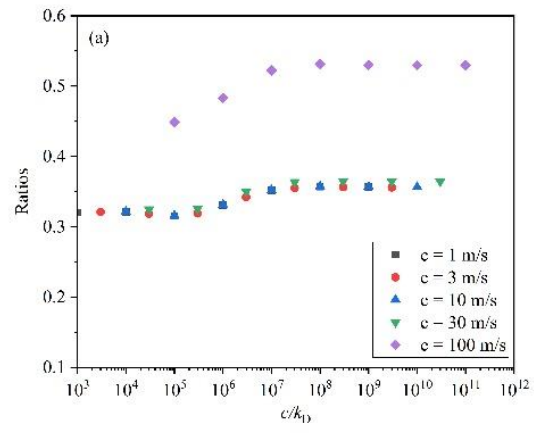

(a)

(b)

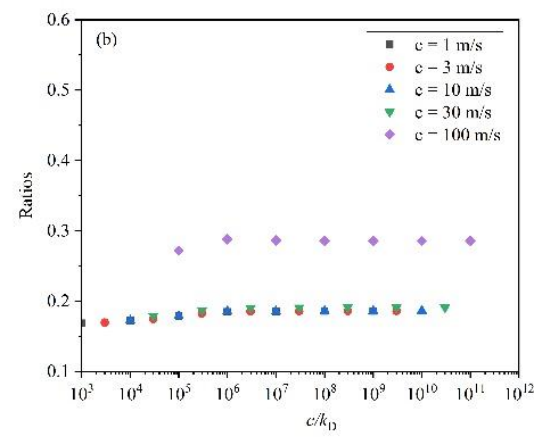

Figure 16. Dependence of stress ratio $\left(\tau / \sigma^{\prime}\right)_{\max }$ on $c / k_{D}$ at different depths: (a) point $\mathrm{A}, z=0.7$ $\mathrm{m}$, (b) point $\mathrm{B}, z=1.2 \mathrm{~m}$

To summarise these results, Fig. 16 shows the maximum absolute value of the stress ratio plotted against $c / k_{D}$; this is independent of the load speed when plotted in this way, except for $20 c=100 \mathrm{~m} / \mathrm{s}$, when the Rayleigh wave speed of the saturated ground $(\sim 112 \mathrm{~m} / \mathrm{s})$ is approached 
1 and the calculated stress ratios, especially at shallow depths, become large. For the depth of $z$

$2=0.7 \mathrm{~m}$ the ratio increases between $c / k_{D}$ of $10^{5}$ and $10^{8}$; for $z=1.2 \mathrm{~m}$ the increase occurs

3 between $c / k_{D}$ of $10^{3}$ and $10^{6}$.

\section{$4 \quad 4.4$ Effect of thickness of soil layer}

5 In the previous analyses, the depth of the saturated layer was $20 \mathrm{~m}$ (Fig. 7). This section is

6 investigates the sensitivity of the results to the depth $H$ of the lower boundary to the saturated

7 soil layer in the model. The total size of the model was kept the same (see Fig. 7), and the

8 depth $H$ to the bottom drainage layer was varied by changing the permeability of the lower

9 part of the model. Results are presented for a constant load speed of $10 \mathrm{~m} / \mathrm{s}$, and five different

10 values of Darcy permeability (ranging from $k_{D}=10^{-3} \mathrm{~m} / \mathrm{s}$ to $10^{-7} \mathrm{~m} / \mathrm{s}$ ). Four different

11 saturated layer thicknesses (i.e., depths to the lower high-permeability layer; $H=20 \mathrm{~m}, 10 \mathrm{~m}$,

$125 \mathrm{~m}$ and $2.5 \mathrm{~m}$ ). The excess pore pressure at the lower boundary was effectively set to zero in 13 each case.
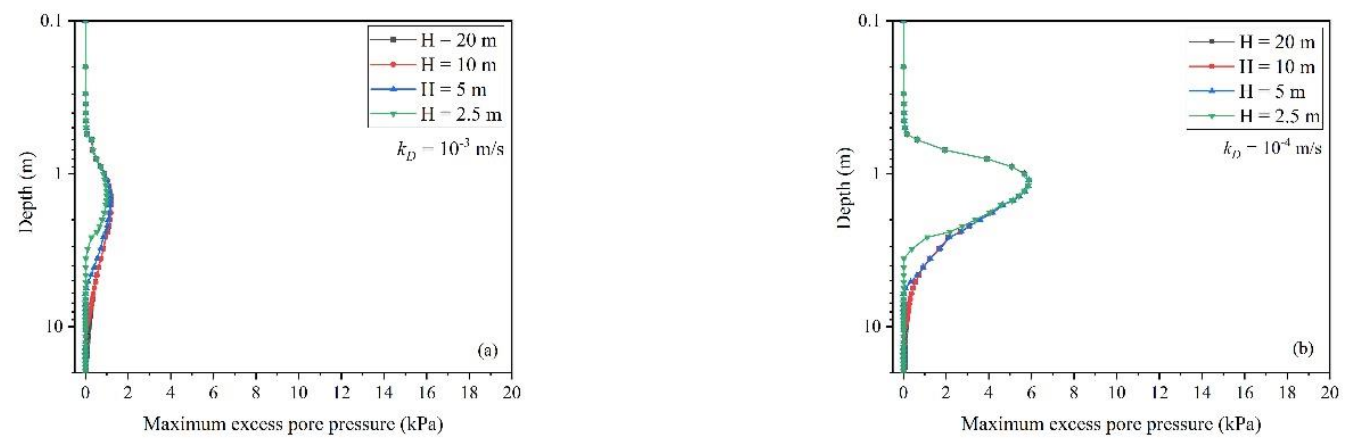


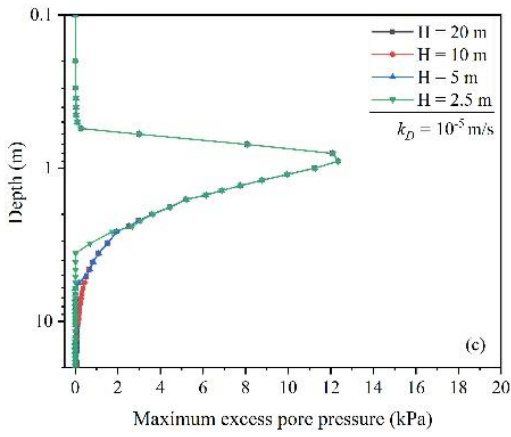

(c)

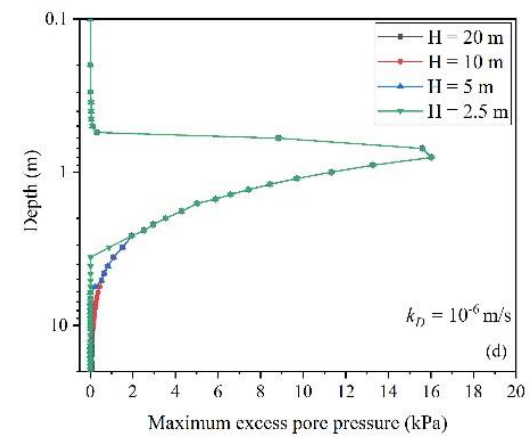

(d)

4 Figure 17. Distribution of pore pressure with depth for different depths of saturated soil layer

5 for a load speed $c=10 \mathrm{~m} / \mathrm{s}$. (a) $k_{D}=10^{-3} \mathrm{~m} / \mathrm{s}$; (b) $k_{D}=10^{-4} \mathrm{~m} / \mathrm{s}$; (c) $k_{D}=10^{-5} \mathrm{~m} / \mathrm{s}$;

$6 \quad$ (d) $k_{D}=10^{-6} \mathrm{~m} / \mathrm{s}$

Fig. 17 shows the distribution of maximum excess pore pressure with depth for the different layer depths; these are the values occurring when the load was directly above the observation point. Each graph is for a different values of soil permeability. The depth of the saturated layer only has a noticeable effect when it is reduced to less than $10 \mathrm{~m}$. Especially at $k_{D}=10^{-3} \mathrm{~m} / \mathrm{s}$, a shorter distance to the lower drainage layer results in lower pore pressures at every depth below about $1 \mathrm{~m}$. However, at these high permeabilities the excess pore pressures are in any case small, and the variation at a given depth is rarely if ever more than $1 \mathrm{kPa}$.

Thus it can be concluded that, provided the saturated soil layer is thicker than $2.5 \mathrm{~m}$, the depth to a drainage layer below it has no practical significance for excess pore water pressure generation during the application of a moving load. Either the soil is of such low permeability that the depth to the drainage layer would need to be much less than $2.5 \mathrm{~m}$ to make a difference, or the soil is of such a high permeability that the excess pore water pressures are small enough to be neglected.

20 This is confirmed by Fig. 18, in which the maximum pore pressures at depths of $0.7 \mathrm{~m}$ and $1.2 \mathrm{~m}$ are plotted against $c / k_{D}$ for all drainage layer depths $H$ investigated. The maximum 
1 excess pore pressure shows the same increase for all values of $H$ when the soil permeability is

2 decreased. The critical value of $c / k_{D}$ (above which the maximum excess pore water pressure

3 shows no further increase with $c / k_{D}$ ) is also the same at each depth, irrespective of the value

4 of $H$. Thus it can be concluded that, within the ranges investigated, the depth to the lower

5 drainage layer has no practically significant effect on the excess pore pressure response in a

6 saturated soil.

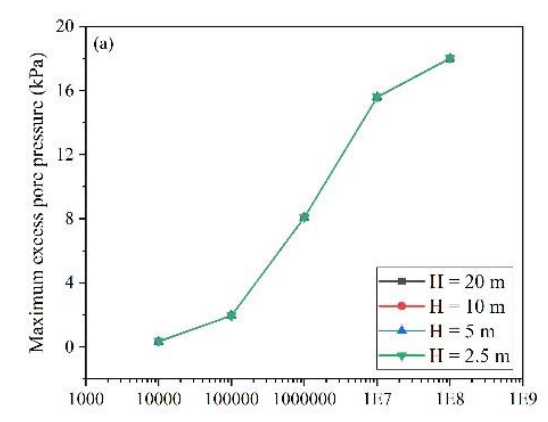

$c / k_{p}$

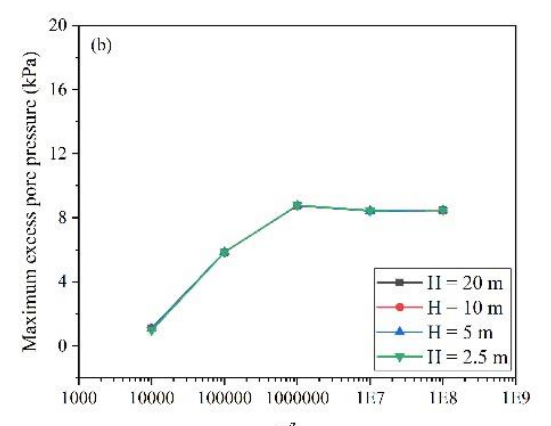

$c / k_{D}$
7

8

9 Figure 18. Development of pore pressure for different thicknesses of saturated layer: (a)

10 point $\mathrm{A}, z=0.7 \mathrm{~m}$, (b) point $\mathrm{B}, z=1.2 \mathrm{~m}$

\subsection{Effect of soil stiffness}

In this section, variations in the soil stiffness (shear modulus, $G$ ) are considered. The

13 thickness of the saturated layer $H$ was fixed at $20 \mathrm{~m}$, and the load speed was $10 \mathrm{~m} / \mathrm{s}$. Three

14 values of soil stiffness were used; $G=40 \mathrm{MPa}$ as in the initial analyses, $G=13.3 \mathrm{MPa}$ (i.e.,

$15 \div 3$ ) and $G=120 \mathrm{MPa}\left(\right.$ i.e., $\times 3$ ). The soil Darcy permeability was varied from $k_{D}=10^{-3} \mathrm{~m} / \mathrm{s}$ to $1610^{-7} \mathrm{~m} / \mathrm{s}$.

17 Fig. 19 shows the time history of excess pore pressure at a depth $z=0.7 \mathrm{~m}$ for the three

18 different soil stiffnesses and a soil permeability of $10^{-5} \mathrm{~m} / \mathrm{s}$. The excess pore pressures

19 generated reduce as the soil becomes stiffer. This is consistent with the Terzaghi 
1 consolidation model, with a stiffer soil consolidating (i.e., excess pore pressures dissipating)

2 more quickly than a less stiff material because the associated volume changes are smaller.

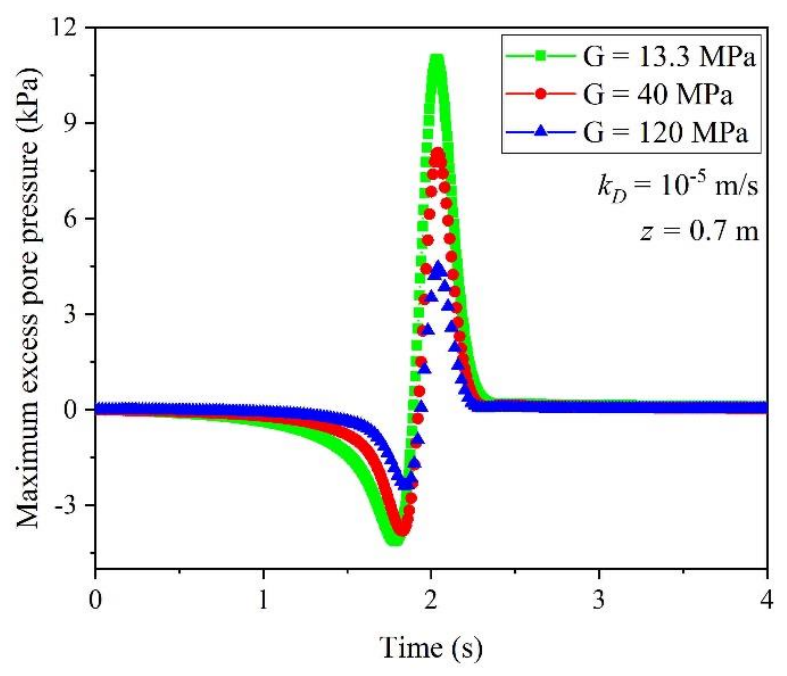

3

4 Figure 19. Time history curve of pore pressure for different soil stiffnesses at point $\mathrm{A}, z=$

$5 \quad 0.7 \mathrm{~m}$

6 Fig. 20 shows the maximum excess pore pressure as a function of depth for these three

7 values of soil stiffness, which has been normalised in each case by the maximum value. As

8 indicated in Fig. 19, the maximum excess pore pressure reduces as the stiffness is increased.

9 However, the maximum always occurs at a depth $z=0.9 \mathrm{~m}$, and when normalised to their

10 maximum values the results are independent of soil stiffness. Moreover, in all cases the

11 excess pore water pressure has fallen to zero at $z=10 \mathrm{~m}$, as in the earlier analyses. This is

12 consistent with Terzaghi consolidation theory: while the soil stiffness will affect the amount

13 of consolidation (volume change) and hence the time for consolidation to occur, it will not

14 affect the relative shape of the excess pore pressure distribution at a given time (known as an

15 isochrone) in the way that a change in the soil permeability does. 


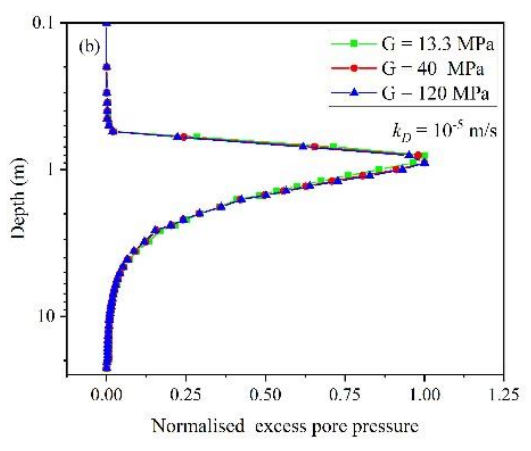

2 Figure 20. Normalised distributions of excess pore pressure with depth for soil stiffnesses $G$ $3=13.3 \mathrm{MPa}, 40 \mathrm{MPa}$, and $120 \mathrm{MPa}$

$4 \quad$ The maximum excess pore pressure for these three stiffnesses is plotted against $c / k_{D}$ in

5 Fig. 21. The soil stiffness influences the excess pore pressures generated quite significantly, 6 but does not alter the critical value of $c / k_{D}$.

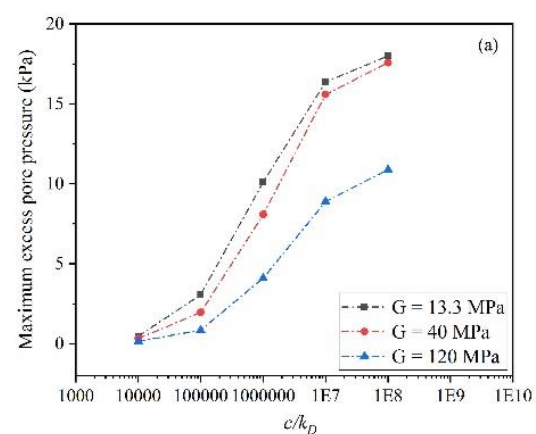

(a)

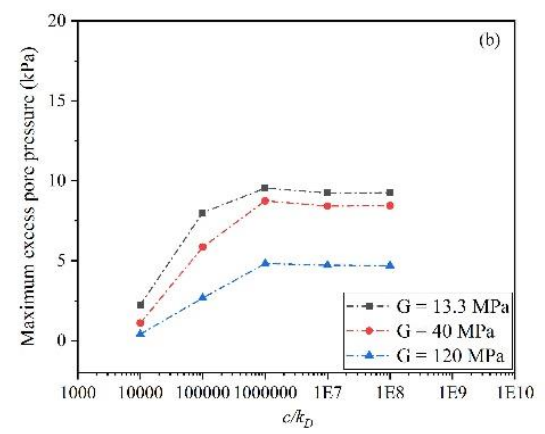

(b)

9 Figure 21. Development of pore pressure in soils of different stiffness: (a) point A, $z=0.7 \mathrm{~m}$,

10 (b) point $\mathrm{B}, z=1.2 \mathrm{~m}$

\section{Numerical results for moving train loads}

In this section, the same track-embankment-ground model is used to calculate the dynamic

14 response induced by the axles of a train. In this case, the lateral load distribution below the

15 ballasted track has been taken into account using the approach proposed by Takemiya [31] to

16 approximate the lateral variation in vertical stress beneath the sleepers. 
1 To investigate the dynamic response of the soil to the succession of loads associated with a

2 moving train, loads corresponding to a $1 / 4$ car of a Velaro train (Table 5) was used. Seven

3 different loading regimes were considered; a single axle, a single bogie (two axles), a single

4 vehicle (4 axles), two vehicles, four vehicles, eight vehicles and 16 vehicles. The analyses

5 were carried out initially for a load speed of $10 \mathrm{~m} / \mathrm{s}$ and soil Darcy permeability of $10^{-6} \mathrm{~m} / \mathrm{s}$.

6 Table 5. Parameters for the Velaro train [1]

\begin{tabular}{ll}
\hline Parameter & Value \\
\hline Mass of a car body & $45000 \mathrm{~kg}$ \\
Mass of a bogie & $3600 \mathrm{~kg}$ \\
Mass of the wheelsets on a bogie & $1700 \mathrm{~kg}$ \\
Vehicle length $L$ & $24.8 \mathrm{~m}$ \\
Bogie centre spacing $b$ & $17.4 \mathrm{~m}$ \\
Wheel spacing $a$ & $2.5 \mathrm{~m}$ \\
\hline
\end{tabular}

7

\subsection{Different lengths of train}

Fig. 22 shows the time history curves of excess pore pressure at a depth $z=0.7 \mathrm{~m}$ in

10 response to the different train loads. The short distance between the two wheels means that

11 the passage of a bogie is seen at this depth as essentially a single loading event (Fig. 22(a)):

12 this is consistent with [32]. The passage of a single bogie gives rise to a small residual excess

13 pore pressure. For a single vehicle (Fig. 22(b)), the pore pressure accumulated after the first

14 bogie has passed starts to dissipate during the rest period between two bogies; the pair of

15 bogies between four vehicles results a more significant accumulation of pore pressure than

16 for a single bogie. Fig. 22(c,d) shows that the final residual excess pore pressure increases

17 slightly during the passage of each vehicle, reaching a maximum after the last bogie of the

18 train has passed. 


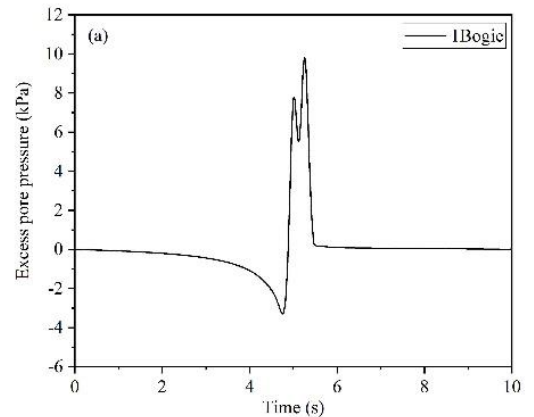

1

2

(a)

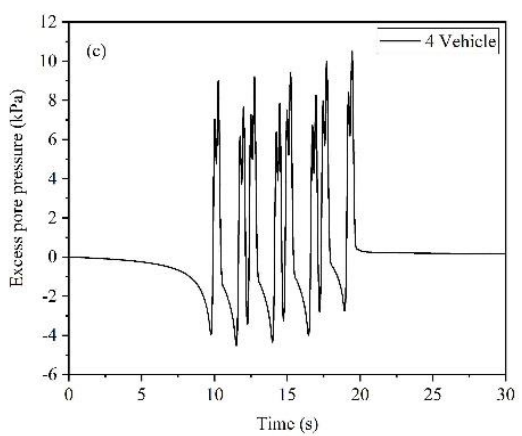

(c)

5

6

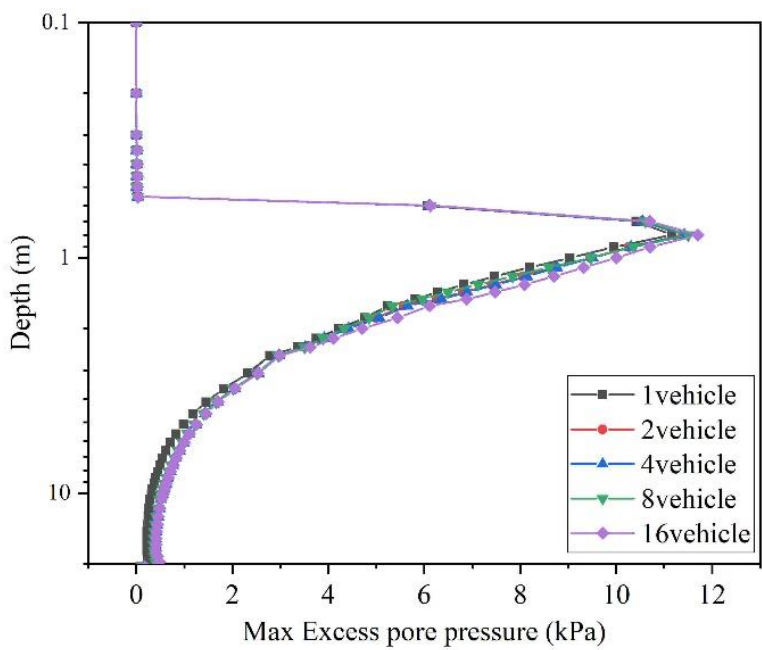

7

8 Figure 23. Maximum pore pressure during the passage of the train plotted against depth for

9 different train lengths

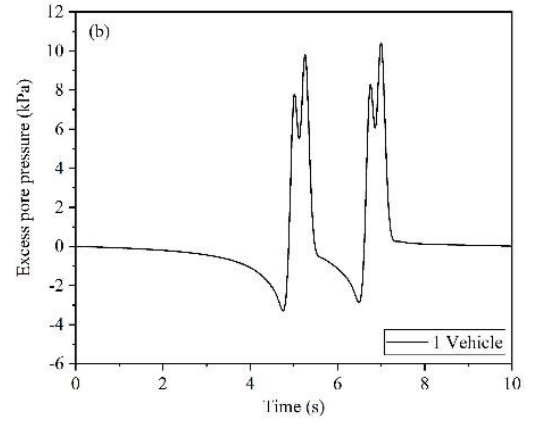

(b)

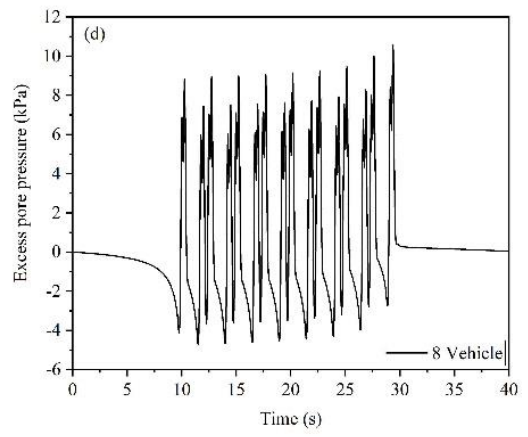

(d)
Figure 22. Time history curves of pore pressure at point A $(z=0.7 \mathrm{~m})$ for different train

lengths. (a) single bogie, (b) single vehicle, (c) four vehicles and (d) eight vehicles

7 
2 Fig. 23 shows the distribution of maximum excess pore pressure against depth for the

3 different lengths of train. This corresponds to the excess pore pressure when the last axle

4 arrives at the observation position. Thus, during the passage of the whole train, the maximum

5 excess pore pressure is $\sim 12 \mathrm{kPa}$. When there are more vehicles in the train, the excess pore

6 pressure at each depth increases slightly.

\section{$7 \quad 5.2$ Effect of permeability and velocity}

8

Fig. 24 shows the excess pore pressure time histories at a depth $z=0.7 \mathrm{~m}$ for a two-car

9 train with different values of soil permeability.

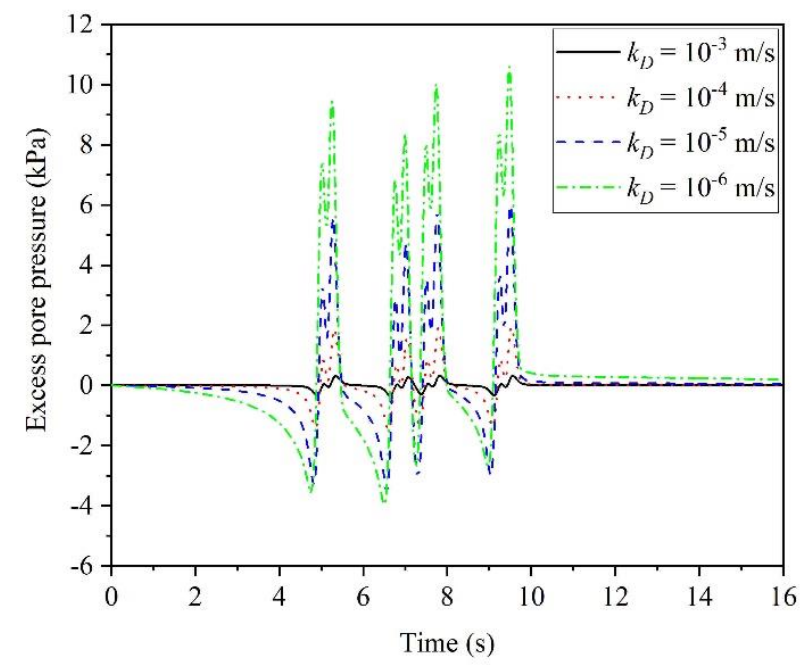

10

11 Figure 24. Time histories of pore pressure for a two-car train at different permeabilities at

12 point $\mathrm{A}$, a depth $z=0.7 \mathrm{~m}$

13 As with the results for the single point load shown in Fig. 8, the excess pore pressure is

14 very small when the soil permeability is high $\left(10^{-3} \mathrm{~m} / \mathrm{s}\right)$. When the soil permeability is very

15 low, the dynamic pore pressure response is much larger and broadly symmetrical. As also

16 seen in Section 4, at intermediate values of permeability the curve is highly asymmetrical,

17 with negative pore pressures being generated in advance of the train. 


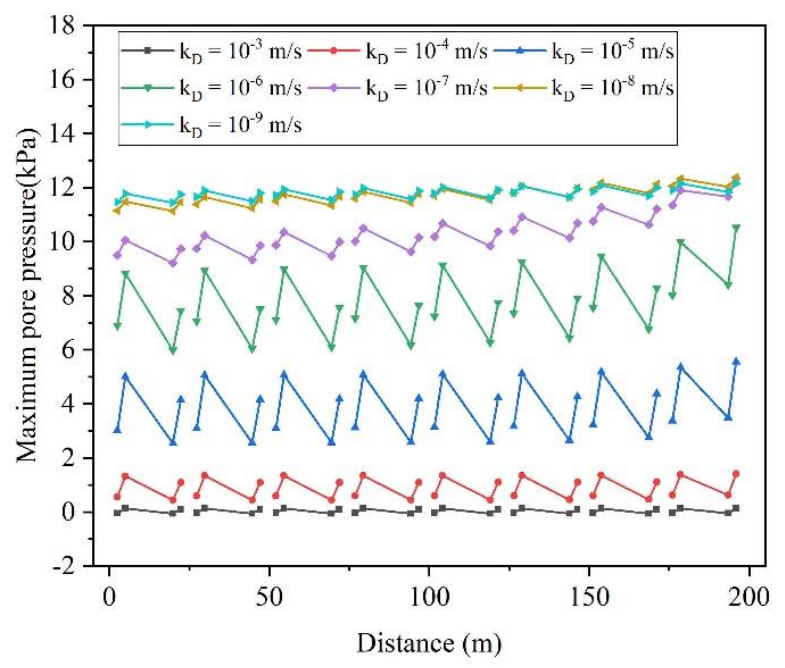

1

2 Figure 25. Maximum pore pressure at $z=0.7 \mathrm{~m}$ under each wheelset during the passage of an

3 eight-car train

To illustrate the evolution of maximum pore pressures during the passage of the train, Fig.

525 shows the maximum excess pore pressure at a depth $z=0.7 \mathrm{~m}$ when each wheel is directly

6 above the observation point, during the passage of an eight-car train. In the graph, the points

7 are connected for wheelsets within the same vehicle. Fig. 25 shows clearly that the maximum

8 excess pore pressure increases as the soil permeability decreases; as before, this is consistent

9 with the potential for pore pressure dissipation during loading being reduced. The maximum

10 excess pore pressures after the last axle has passed are quite similar for permeabilities of $10^{-7}$,

$1110^{-8}$ and $10^{-9} \mathrm{~m} / \mathrm{s}$. This reflects the critical value of $c / k_{D}$, and indicates a critical value of $c / k_{D}$

12 of $10^{8}$, the same as for the single moving load. Thus the critical value of $c / k_{D}$ is a function of

13 the soil, and does not depend on the loading pattern. For intermediate values of permeability,

14 particularly $10^{-5}$ and $10^{-6} \mathrm{~m} / \mathrm{s}$, there is a significant increase in maximum pore pressure during

15 the passage of each bogie pair, as seen in Fig. 24.

16 


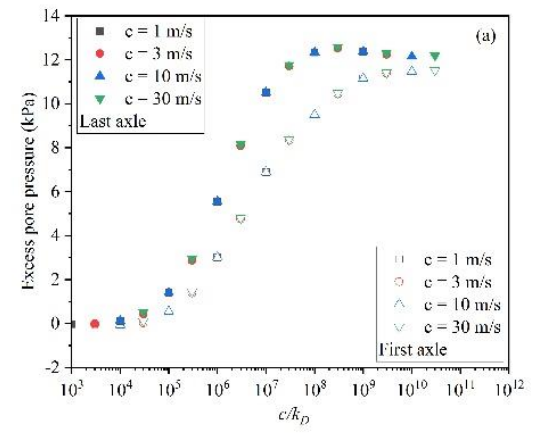

2

3

4

5

6 $c / k_{D}$, the result reduces to around $0.7 \mathrm{kPa}$.

\section{Summary and conclusions}

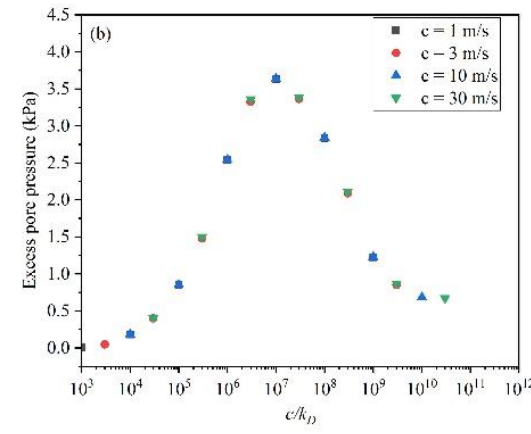

(b)

Figure 26. (a) Maximum pore pressure at point $\mathrm{A}(z=0.7 \mathrm{~m})$ beneath first and last axles of an eight-car train. (b) Difference between maximum pressures at last and first axles.

To show the dependency of these results on permeability, Fig. 26(a) plots the excess pore pressure under the first axle and the last axle of an eight-car train against $c / k_{D}$; presented in this way, these results are independent of the train speed. The results for a speed of $100 \mathrm{~m} / \mathrm{s}$ (not shown), are slightly greater in each case owing to the proximity to the critical speed.

Fig. 26(b) plots the increase in excess pore pressure associated with the passage of an eightcar train against $c / k_{D}$; these results are the differences between the pressures for the last and first axles in Fig. 26(a). The resulting curve is again independent of the train speed. In this case, a peak occurs at a value of $c / k_{D}$ of around $10^{7}$ for this depth. With further increases in

Using Biot's theory of wave propagation in poro-elastic media, a 2.5D finite element model has been presented in $u-w$ format. The model has been used to carry out a fully coupled, wavenumber/frequency domain analysis of the dynamic response of saturated ground to moving steady train loads on a railway track on a shallow embankment. The approach was validated by comparing results with semi-analytical solutions for both simple elastic and saturated poro-elastic media. The model has then been used to investigate the 
1 excess pore pressure response to a single moving load and a moving train of loads

2 considering variations in the soil permeability, load or train speed, depth of saturated layer,

3 and soil stiffness. At train speeds below the speed of Rayleigh waves in the soil, the main

4 findings are as follows:

1. In a saturated soil of a particular stiffness, the load speed $c$ and the soil Darcy

6 permeability $k_{D}$ together determine the extent to which excess pore pressures build up during passage of the load, at any given depth. If the ratio $c / k_{D}$ is less than $10^{4}$, the soil can be viewed as highly permeable in relation to the load speed and almost no excess pore pressure is developed.

2. When the thickness of the saturated soil layer (i.e. the depth to a lower, zero pore pressure boundary) is greater than $2.5 \mathrm{~m}$, this depth has only a limited impact on the excess pore pressure response for the range of soil stiffnesses considered.

3. The variation in the maximum stress ratio, $\left(\tau / \sigma^{\prime}\right)_{\max }$, with permeability depends on the depth under consideration. At a shallow depth, there is no monotonic relationship and there may be an optimum soil permeability from the point of view of minimizing the stress ratio. At slightly greater depths, the stress ratio is likely to increase if the permeability reduces below a certain value.

4. The soil stiffness affects the magnitude of the excess pore pressure response, with higher values of stiffness leading to lower excess pore pressures as a greater part of the stress is carried by the soil skeleton. However, when normalised relative to the maximum value, the distribution of excess pore pressure with depth is unaffected.

5. During the passage of a train, the excess pore pressure under each axle increases slightly with each vehicle passage and reaches a maximum when the last bogie of the train passes. 
6. For the passage of a single axle or a train of axle loads, there is a critical value of $c / k_{D}$, above which the maximum pore pressure reaches a constant value. Increasing $c / k_{D}$ above this critical value does not then have any further impact on the excess pore pressure generated. Below the critical value, the pore pressure accumulated during the passage of a train depends on $c / k_{D}$ but is otherwise independent of the load speed.

7. The accumulation of excess pore pressure during the passage of a bogie pair is greatest for intermediate values of $c / k_{D}$.

All data published in this paper are openly available from the University of Southampton repository at https://doi.org/10.5258/SOTON/D0963.

\section{Acknowledgements}

Financial support is acknowledged from the National Natural Science Foundation of China (Grant No.51678524), Advanced Newton Fellowship of Royal Society, UK (NA140060) and Distinguished Young Scientist Program of Zhejiang Province (LR16E080002), and sponsorship from Chinese Scholarship Council. The work has also been supported by the EPSRC under the programme grant EP/M025276/1, 'The science and analytical tools to design long life, low noise railway track systems (Track to the Future)'.

\section{References}

[1] Milne D, Le Pen L, Thompson D, and Powrie W. "Properties of train load frequencies and their applications," J Sound Vib, 2017; 397:123-140.

[2] Biot M. A, "Theory of propagation of elastic waves in a fluid - saturated porous solid. I. Low frequency range," J. Acoust. Soc. Am, 1956; 28(2): 179-191.

[3] Zienkiewicz O. and Shiomi T. "Dynamic behaviour of saturated porous media; the generalized Biot formulation and its numerical solution," Int J Numer Anal Met, 1984; 8(1): 71-96.

[4] Siddharthan R, Zafir Z, and Norris G. M. "Moving load response of layered soil. I: Formulation," J Eng Mech, 1993; 119(10): 2052-2071.

[5] Lu J.-F. and Hanyga A. "Fundamental solution for a layered porous half space subject to a vertical point force or a point fluid source," Comput Mech, 2005; 35(5): 376-391.

[6] Lu J.-F. and Jeng D.-S. "A half-space saturated poro-elastic medium subjected to a moving point load," Int J Solids Struct, 2007; 44(2): 573-586. 
[7] Xu B, Lu J.-F, and Wang J.-H. "Dynamic response of an infinite beam overlying a layered poroelastic half-space to moving loads," J Sound Vib, 2007; 306(1-2): 91110.

[8] Xu B, Lu J.-F, and Wang J.-H. "Dynamic response of a layered water-saturated half space to a moving load," Comput Geotech, 2008; 35(1): 1-10.

[9] Gao G.-Y, Chen Q, He J, and Liu F. "Investigation of ground vibration due to trains moving on saturated multi-layered ground by 2.5 D finite element method," Soil Dyn Earthq Eng, 2012; 40(87-98.

[10] He C, Zhou S, Di H, and Shan Y. "A 2.5-D coupled FE-BE model for the dynamic interaction between saturated soil and longitudinally invariant structures," Comput Geotech, 2017; 82(211-222.

[11] He C, Zhou S, Guo P, Di H, and Xiao J. "Dynamic 2.5-D Green's function for a point load or a point fluid source in a layered poroelastic half-space," Eng Anal Bound Elem, 2017; 77:123-137.

[12] Yuan W, Cai Y. Q, Shi L, and Cao Z. G. "Study of vibration-isolation efficiency of open trench in saturated ground by 2.5D finite element method," Rock \& Soil Mech, 2013; 34(7): 2111-2118.

[13] Lefeuve-Mesgouez G. and Mesgouez A. "Ground vibration due to a high-speed moving harmonic rectangular load on a poroviscoelastic half-space," Int J Solids Struct, 2008; 45(11-12): 3353-3374.

[14] Jin B. "Dynamic response of a poroelastic half space generated by high speed road.," Chin Quart Mech, 2004; 25:168-74.

[15] Jin B, Yue Z, and Tham L. "Stresses and excess pore pressure induced in saturated poroelastic halfspace by moving line load," Soil Dyn Earthq Eng, 2004; 24(1): 25-33.

[16] Cai Y, Sun H, and Xu C. "Response of railway track system on poroelastic half-space soil medium subjected to a moving train load," Int J Solids Struct, 2008; 45(18-19): $5015-5034$.

[17] Cai Y, Sun H, and Xu C. "Three-dimensional analyses of dynamic responses of trackground system subjected to a moving train load," Comp \& Struct, 2008; 86(7-8): 816824.

[18] Theodorakopoulos D. "Dynamic analysis of a poroelastic half-plane soil medium under moving loads," Soil Dyn Earthq Eng, 2003; 23(7): 521-533.

[19] Theodorakopoulos D, Chassiakos A, and Beskos D. "Dynamic effects of moving load on a poroelastic soil medium by an approximate method," Int J Solids Struct, 2004; 41(7): 1801-1822.

[20] Yuan Z, Xu C, Cai Y, and Cao Z. "Dynamic response of a tunnel buried in a saturated poroelastic soil layer to a moving point load," Soil Dyn Earthq Eng, 2015; 77(348359.

[21] Yuan Z, Cai Y, and Cao Z. "An analytical model for vibration prediction of a tunnel embedded in a saturated full-space to a harmonic point load," Soil Dyn Earthq Eng, 2016; 86:25-40.

[22] Di H, Zhou S, He C, Zhang X, and Luo Z. "Three-dimensional multilayer cylindrical tunnel model for calculating train-induced dynamic stress in saturated soils," Comput Geotech, 2016; 80:333-345.

[23] Di H, Zhou S, Luo Z, He C, Xiao J, and Li X. "A vehicle-track-tunnel-soil model for evaluating the dynamic response of a double-line metro tunnel in a poroelastic halfspace," Comput Geotech, 2018; 101: 245-263.

[24] Biot M. A. "Mechanics of deformation and acoustic propagation in porous media," J. Appl. Phys, 1962; 33(4): 1482-1498. 
[25] Liu G. and Jerry S. Q. "A non-reflecting boundary for analyzing wave propagation using the finite element method," Finite Elem Anal Des, 2003; 39(5-6): 403-417.

[26] Takemiya H. and Bian X. "Substructure Simulation of Inhomogeneous Track and Layered Ground Dynamic Interaction under Train Passage," J Eng Mech, 2005; 131(7): 699-711.

[27] Kausel E. and Roesset J. M. "Stiffness matrices for layered soils," B Seismol Soc Am, 1981; 71(6): 1743-1761.

[28] Fayad P. H. "Aspects of the volumetric and undrained behavior of Boston blue clay," Massachusetts Institute of Technology, Doctoral dissertation, 1986.

[29] Sheahan T. C. "An experimental study of the time-dependent undrained shear behavior of resedimented clay using automated stress path triaxial equipment," Massachusetts Institute of Technology, Doctoral dissertation, 1991.

[30] Yin Z.-Y, Xu Q, and Hicher P.-Y. "A simple critical-state-based double-yield-surface model for clay behavior under complex loading," Acta Geotech, 2013; 8(5): 509-523.

[31] Krylov V. V. Noise and vibration from high-speed trains. Thomas Telford, 2001.

[32] Yang L, Powrie W, and Priest J. "Dynamic stress analysis of a ballasted railway track bed during train passage," J Geotech Geoenviron, 2009; 135(5): 680-689. 
1 Appendix A. Expressions for terms in Eq. (7)

$$
\begin{aligned}
& \mathbf{K}_{\mathbf{1}}=\sum_{e} \int_{-1}^{1} \int_{-1}^{1}\left(\mathbf{B}^{*} \mathbf{N}\right)^{\mathbf{T}} \mathbf{D B N}|\mathbf{J}| d \eta d \varsigma \\
& \mathbf{K}_{\mathbf{2}}=\left(\alpha^{2} M\right) \sum_{e} \int_{-1}^{1} \int_{-1}^{1}\left(\mathbf{B}^{*} \mathbf{N}\right)^{\mathbf{T}} \mathbf{m m}^{\mathbf{T}} \mathbf{B N}|\mathbf{J}| d \eta d \varsigma \\
& \mathbf{K}_{\mathbf{3}}=(a M) \sum_{e} \int_{-1}^{1} \int_{-1}^{1}\left(\mathbf{B}^{*} \mathbf{N}\right)^{\mathbf{T}} \mathbf{m m}^{\mathbf{T}} \mathbf{B} \mathbf{N}|\mathbf{J}| d \eta d \varsigma \\
& \mathbf{M}_{\mathbf{1}}=\omega^{2} \rho_{\mathrm{b}} \sum_{e} \int_{-1}^{1} \int_{-1}^{1} \mathbf{N}^{\mathbf{T}} \mathbf{N}|\mathbf{J}| d \eta d \varsigma \\
& \mathbf{M}_{\mathbf{2}}=\mathbf{M}_{3}=\omega^{2} \rho_{f} \sum_{e} \int_{-1}^{1} \int_{-1}^{1} \mathbf{N}^{\mathbf{T}} \mathbf{N}|\mathbf{J}| d \eta d \varsigma \\
& \mathbf{M}_{\mathbf{4}}=\left(\omega^{2} m-i \omega \frac{\rho_{f} g}{k_{D}}\right) \sum_{e}^{1} \int_{-1}^{1} \int_{-1}^{1} \mathbf{N}^{\mathbf{T}} \mathbf{N}|\mathbf{J}| d \eta d \varsigma \\
& \mathbf{L}_{\mathbf{1}}=a M \sum_{e} \int_{-1}^{1} \int_{-1}^{1}\left(\mathbf{B}^{*} \mathbf{N}\right)^{\mathbf{T}} \mathbf{m} \mathbf{m}^{\mathbf{T}} \mathbf{B} \mathbf{N}|\mathbf{J}| d \eta d \varsigma \\
& \mathbf{L}_{\mathbf{2}}=M \sum_{e} \int_{-1}^{1} \int_{-1}^{1}\left(\mathbf{B}^{*} \mathbf{N}\right)^{\mathbf{T}} \mathbf{m} \mathbf{m}^{\mathbf{T}} \mathbf{B} \mathbf{N}|\mathbf{J}| d \eta d \varsigma \\
& \widetilde{\boldsymbol{F}}^{s}=\sum_{e} \int \boldsymbol{N}^{T} \tilde{\tilde{f}}|\mathbf{J}| d \eta \\
& \widetilde{\boldsymbol{F}}^{f}=\sum_{e} \int \boldsymbol{N}^{T} \tilde{\bar{q}}|\mathbf{J}| d \eta
\end{aligned}
$$

2 where the integrals are over local coordinates $\eta$ and $\varsigma$, and 
$1 \quad \mathbf{D}=\left(\begin{array}{cccccc}\lambda+2 \mu & \lambda & \lambda & 0 & 0 & 0 \\ \lambda & \lambda+2 \mu & \lambda & 0 & 0 & 0 \\ \lambda & \lambda & \lambda+2 \mu & 0 & 0 & 0 \\ 0 & 0 & 0 & \mu & 0 & 0 \\ 0 & 0 & 0 & 0 & \mu & 0 \\ 0 & 0 & 0 & 0 & 0 & \mu\end{array}\right) \quad \mathbf{B}=\left(\begin{array}{ccc}-i \xi_{x} & 0 & 0 \\ 0 & \frac{\partial}{\partial y} & 0 \\ 0 & 0 & \frac{\partial}{\partial z} \\ \frac{\partial}{\partial y} & -i \xi_{x} & 0 \\ 0 & \frac{\partial}{\partial z} & \frac{\partial}{\partial y} \\ \frac{\partial}{\partial z} & 0 & -i \xi_{x}\end{array}\right) \quad \mathbf{m}=\left(\begin{array}{l}1 \\ 1 \\ 1 \\ 0 \\ 0 \\ 0\end{array}\right)$

$2 \quad|\mathbf{J}|=\left|\begin{array}{ll}\sum_{i=1}^{4} \frac{\partial \mathbf{N}_{\mathrm{i}}}{\partial \eta} y_{i}^{e} & \sum_{i=1}^{4} \frac{\partial \mathbf{N}_{\mathrm{i}}}{\partial \xi} y_{i}^{e} \\ \sum_{i=1}^{4} \frac{\partial \mathbf{N}_{\mathrm{i}}}{\partial \eta} z_{i}^{e} & \sum_{i=1}^{4} \frac{\partial \mathbf{N}_{\mathrm{i}}}{\partial \xi} z_{i}^{e}\end{array}\right|$ 